\title{
Analysis of heat and fluid flow around two co-rotating side-by-side cylinders
}

\author{
RAHIM HASSANZADEH*iD and MOHSEN DARVISHYADEGARI \\ Faculty of the Mechanical Engineering, Urmia University of Technology, Urmia, Iran \\ e-mail: r.hassanzadeh@uut.ac.ir; mdarvishyadegari68@gmail.com
}

MS received 13 March 2018; revised 1 November 2018; accepted 20 February 2019; published online 6 April 2019

\begin{abstract}
Analysis of the heat and fluid flow around two co-rotating side-by-side cylinders is the subject of this numerical study which is done at constant Reynolds and Prandtl numbers of 200 and 7.0, respectively. Both cylinders rotate in the counterclockwise direction with an identical rotating speed (RS) in the range from 0 to 4. On the other hand, several gap spaces between the rotating cylinders such as $\mathrm{G} / \mathrm{D}=1.5,2.0$, and 3.0 are considered in the present study. The obtained results are validated against the available data in the open literature. Many different results have been reported in this investigation. It is observed that co-rotating the cylinders deforms the wake region downstream of both cylinders which the vortex strength of the lower cylinder against the rotation is stronger than that of the upper cylinder. On the other hand, co-rotating the cylinders develops a negative lift force on both cylinders. Finally, it was concluded that rotating the side-by-side cylinders reduces the heat transfer rate between the fluid flow and cylinders in general. At whole RS and G/D values, the heat transfer rate of the upper cylinder is realized to be less than that of the lower cylinder.
\end{abstract}

Keywords. Forced convection; co-rotating cylinders; side-by-side cylinders; fluid rotating zone.

\section{Introduction}

To date, the flow around the bluff bodies has been of interest among the researchers due to its serious applications in practical engineering. Among the bluff bodies, the flow around the cylinders has historically found considerable attention for numerous researchers due to its fundamental flow nature. A general review of the published works shows that the flow around a single cylinder in the free-stream and near a wall [1-12] has been studied in terms of the vortex shedding phenomenon, active and passive flow control, and wake structure in laminar, transitional, and turbulent regimes. Beyond the single cylinder, the flow around the dual cylinders in various arrangements such as the side-byside and tandem [13-21] has been attractive among the researchers not only using different numerical studies but also with various experiments to reveal the interaction level between the cylinders at different gap spaces. The flow around a rotating cylinder with constant $\mathrm{RS}$ has been introduced as a mechanism to control the wake flow and surface temperature andlor heat flux. In such problems, the flow behavior becomes more complex due to an additional issue of the rotation in comparison to the stationary cylinder. In the past decade, several studies have been performed regarding the rotating cylinders. For instance, Shaafi et al [22] investigated the interaction between a laminar

*For correspondence boundary layer developed on a flat wall and a rotating cylinder with clockwise and counterclockwise rotation at $\operatorname{Re}=100$. They considered the non-dimensional gap space between the cylinder and flat wall in the range from 0.1 to 3 . They showed that clockwise rotation amplitudes the integral parameters with the counterclockwise attenuates. In a numerical work, Rana et al [23] studied the gust response of a rotating cylinder in the vortex suppression regime under $2 \leq \mathrm{RS} \leq 5$ and $\mathrm{Re}=140$ in terms of the lift and drag coefficients, temperature field, and the Nusselt number. In another numerical study, Bayat and Rahimi [24] conducted the flow around a rotating vertical cylinder at $\operatorname{Re}=1,5,10$, 15 , and 20 with $\operatorname{Pr}=0.7$. They predicted the location of the cylinder stagnation point as a function of the Grashof and Richardson numbers. Flow around a rotating cylinder embedded in a channel filled with the power-law fluid has been presented by Thakur et al [25] at $0.001 \leq \mathrm{Re} \leq 40$. They stated that for a constant Re number, the wake length increases with the increasing degree of shear-thinning behavior and the degree of symmetry and in this situation, the flow transits to an unsteady regime. Naik et al [26] demonstrated the flow pattern around a rotating elliptic cylinder at $\mathrm{Re}=100$. They revealed that the lift coefficient increases with increasing RS and decreases with increasing the cylinder aspect ratio. In addition, the drag coefficient decreases with increasing the aspect ratio up to 0.7 and beyond it, increases. Hassanzadeh [27] investigated the effects of unsteady flow generated by a rotating cylinder on 
the cooling mechanism of a hot plate. The value of the Reynolds and Prandtl numbers was 200 and 7.0, respectively and computations were carried out at different RS and G/D values. It was concluded that the maximum heat transfer rate between the generated unsteady flow and the hot plate occurs at $\mathrm{G} / \mathrm{D}=0.5$ at each individual $\mathrm{RS}$ value. Convective heat transfer and fluid flow of two counter-rotating cylinders in the tandem arrangement was the subject of a research work presented by Darvishyadegari and Hassanzadeh [28]. They performed their research at $\mathrm{Re}=200$ and RS in the range between the 0 and 4 . Several results have been reported in their works. For example, for both upstream and downstream cylinders, it was shown that with rotation the cylinders, the negative pressures show a tendency to become dominant all over the surface, and with increasing RS, this negative pressure enhances considerably. In addition, at low RS values, the half-upper side of the upstream cylinder dissipates a higher amount of the heat into the surrounding fluid in comparison to the half-lower side, and vice versa for the downstream cylinder. However, at higher RS values, due to dominating the fluid-rotating zone around both upstream and downstream cylinders, it seems that all points on each cylinder have identical roles in the heat transfer process. In another work by the same authors [29] regarding the heat and fluid flow around two co-rotating tandem cylinders, it has been demonstrated that a uniform Nusselt number distribution can be observed on both cylinders regardless of the gap space between them. Analysis of convective heat transfer and flow nature around two counter-rotating side-by-side cylinders is the subject of a research work performed by Darvishyadegari and Hassanzadeh [30]. They examined several gap spaces and rotating speeds at constant Reynolds number of 200. They concluded that counter-rotating of two side-by-side cylinders suppresses the wake of both cylinders at low RS and eliminated it at high RS. In addition, the rate of the heat transfer for both side-by-side cylinders reduces considerably with increasing RS in counter-rotating scenario.

The problem of the heat transfer around the rotating cylinder is also the subject of some studies. For instance, Selimefendigil et al [31] presented the mixed convection of the nanofluid in a square enclosure. They showed that the Nusselt number enhances with the rotating speed. Kareem and Gao [32] investigated the mixed heat transfer and turbulent flow around a rotating cylinder in a lid-driven cavity at $\operatorname{Re}=5000,10,000,20,000$, and 30,000 and $\mathrm{RS}$ values in the range from 0 to 10 . They reported that the heat and fluid flow affected by both Re and RS. In return, the Nusselt number enhances as a function of the Re and RS. Similar results have been stated by Khanafer and Aithal [33]. In another numerical research, Selimefendigil and Öztop [34] considered the mixed convection of two rotating cylinders in an enclosure filled with various nanofluids. It was demonstrated that for each individual nanofluids, the mean Nusselt number enhances and diminishes under the counterclockwise and clockwise rotation, respectively.
Further examinations of the open literature show that heat and fluid flow around two co-rotating side-by-side cylinders has not been studied to date according to the authors' knowledge. Flow around two rotating side-by-side cylinders is the subject of several engineering applications such as screw compressors and pumps. Performing of this study is in the direction of these engineering applications at which the effects of several non-dimensional parameters such as RS and G/D on the flow behavior and heat transfer have been studied in detail. It is hoped that the obtained results arouse interest among the engineers in various industries.

\section{Problem description and solution method}

In the present study, in order to compute the heat and fluid flow around two co-rotating side-by-side cylinders, in accordance with similar studies published previously, a two-dimensional rectangular flow domain is considered as in figure 1. The physical flow domain has dimensions of $31 \mathrm{D}$ in stream-wise and $21 \mathrm{D}+\mathrm{G}$ in vertical directions. The centers of the cylinders are set at $10.5 \mathrm{D}$ from the incoming section and the distance between the center of each cylinder and the nearest horizontal boundary is also 10.5D. Furthermore, the coordinate's origin is set at the mid-point of the incoming section. Both cylinders rotate in the counterclockwise direction with the same RS in the range from 0 to 4 . On the other hand, three different non-dimensional gap spaces such as $\mathrm{G} / \mathrm{D}=1.5,2.0$, and 3.0 are considered for side-by-side cylinders. All computations are carried out at $\operatorname{Re}=200$ and $\operatorname{Pr}=7.0$. The governing equations of the problem under consideration are as follows;

$$
\begin{gathered}
\nabla \cdot \vec{V}=0 \\
\rho \frac{D \vec{V}}{D t}=-\nabla p+\mu \nabla^{2} \vec{V} \\
\rho c_{p} \frac{D T}{D t}=k \nabla^{2} T
\end{gathered}
$$

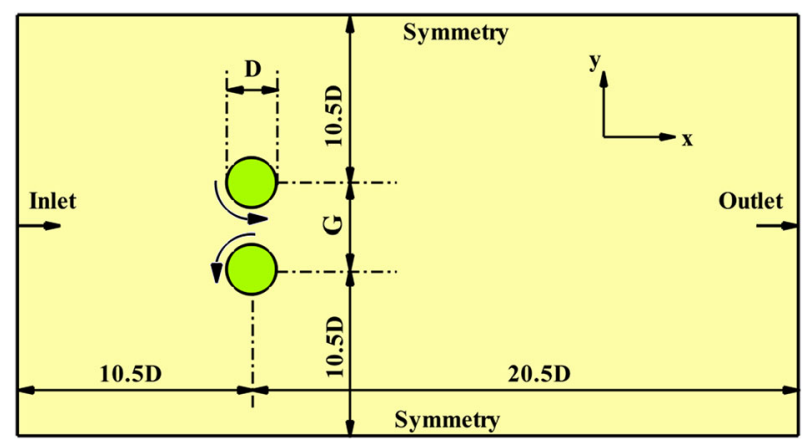

Figure 1. Physical flow domain in this study. 
The spatial terms in governing equations are discretized using the second-order upwind scheme and the temporal derivatives are discretized using a second order accurate method. For coupling the pressure-velocity fields, the SemiImplicit Method for Pressure-Linked Equation (SIMPLE) [35] algorithm is utilized. The discretized equations are solved implicitly using the following boundary conditions;

- At inlet boundary

$$
\begin{aligned}
u & =U \\
v & =0 \\
T & =T_{\infty}
\end{aligned}
$$

- At outlet boundary

$$
\begin{aligned}
& \frac{\partial u}{\partial x}=\frac{\partial v}{\partial x}=0 \\
& \frac{\partial T}{\partial x}=0
\end{aligned}
$$

- At upper and lower boundaries

$$
\begin{aligned}
& \frac{\partial u}{\partial y}=\frac{\partial v}{\partial y}=0 \\
& \frac{\partial T}{\partial y}=0
\end{aligned}
$$

- On the surface of upper and lower cylinders

$$
\begin{aligned}
u & =U(R S) \sin \alpha \\
v & =-U(R S) \cos \alpha \\
T & =T_{s}
\end{aligned}
$$

In addition, the following dimensionless parameters are utilized in the present study;

- Pressure coefficient

$$
C_{p}=\frac{p-p_{\infty}}{0.5 \rho U^{2}}
$$

- Drag coefficient

$$
C_{D}=\frac{F_{D}}{0.5 \rho U^{2} A}
$$

- Lift coefficient

$$
C_{L}=\frac{F_{L}}{0.5 \rho U^{2} A}
$$

- Nusselt number

$$
N u=\frac{-\left.D \frac{\partial T}{\partial r}\right|_{r=R}}{T_{s}-T_{\infty}}
$$

Furthermore, to analyze the rate of the unsteadiness around the two co-rotating side-by-side cylinders, the root mean square (rms) values of the streamwise and vertical velocities are determined as;

$$
\begin{aligned}
& u_{r m s}=\left[(u-\bar{u})^{2}\right]^{0.5} \\
& v_{r m s}=\left[(v-\bar{v})^{2}\right]^{0.5}
\end{aligned}
$$

It should be said that the convergence criteria for all flow variables are set to be less than $10^{-8}$.

\section{Grid size independence study}

In the present study, the multi-block grid systems are applied for computing the discretized governing equations. For this goal, the physical flow domain is firstly divided into several blocks and after that, the non-uniform structured grids are defined for each block at which the size of the grids is finer near the cylinders and by getting away from the cylinders, the size of the grids grows gradually according to a specific function. It should be said that only within one block where the cylinders are located, the unstructured grids with a high resolution is used. In order to find the proper grid resolutions in each $G / D$ value, six various grid resolutions are examined for each G/D. Table 1 shows a sample of examined grid resolutions at $\mathrm{G} / \mathrm{D}=1.5$ and $\mathrm{RS}=0$ for time-averaged lift and drag coefficients and Nusselt number. It is observable that the obtained results do not alter with a further resolution in the grid system at least among the applied six different grid systems. However, the fourth grid system with 46220 control volumes is selected for $\mathrm{G} / \mathrm{D}=1.5$. For the other cases of $\mathrm{G} / \mathrm{D}=2.0$ and 3.0, 52235 and 64254 control volumes are applied, respectively.

\subsection{Validation of the numerical code}

In this study, several attempts have been done in order to ensure that the applied computer code has sufficient accuracy. In the first stage, the numerical results of the stationary side-by-side cylinders are compared with available data [36-38] in the literature. The results of this comparison are shown in table 2. On the other hand, the other comparison has been done for the Nusselt number of a single stationary cylinder which is presented in table 3. Both comparisons show excellent agreements between the results of this computer code and available data.

\section{Results and discussion}

Figure 2 demonstrates time-averaged flow patterns around two co-rotating side-by-side cylinders at different G/D and RS values. In the stationary cases, in accordance with the 
Table 1. Results of grid size independence study for $\mathrm{G} / \mathrm{D}=1.5$ and $\mathrm{RS}=0$.

\begin{tabular}{lccccccc}
\hline Grid No. & Control volume number & $\mathrm{C}_{\mathrm{D}}$ & Error (\%) & $\mathrm{C}_{\mathrm{L}}$ & Error $(\%)$ & $\overline{N u}$ & Error $(\%)$ \\
\hline 1 & 11214 & 1.47 & - & \pm 0.45 & - & 18.10 \\
2 & 15454 & 1.48 & 0.01 & \pm 0.46 & 0.01 & 18.13 \\
3 & 35101 & 1.58 & 0.05 & \pm 0.44 & -0.01 & 18.36 \\
4 & 46220 & 1.52 & -0.03 & \pm 0.46 & 0.01 & 18.08 & -0.12 \\
5 & 68334 & 1.57 & 0.03 & \pm 0.44 & -0.01 & 18.26 \\
6 & 82009 & 1.57 & 0.00 & \pm 0.45 & 0.01 & 18.23 & -0.01 \\
\hline
\end{tabular}

Table 2. Validation of the applied computer code against the available data for two stationary side-by-side cylinders.

\begin{tabular}{|c|c|c|c|c|c|c|}
\hline \multirow[b]{3}{*}{ Author } & \multicolumn{3}{|c|}{$C_{D}$} & \multicolumn{3}{|c|}{$\mathrm{C}_{\mathrm{L}}$} \\
\hline & \multicolumn{3}{|c|}{ G/D } & \multicolumn{3}{|c|}{$\mathrm{G} / \mathrm{D}$} \\
\hline & 1.5 & 2.0 & 3.0 & 1.5 & 2.0 & 3.0 \\
\hline Ding et al [36] & $1.52 \& 1.54$ & N.A & 1.54 & $0.43 \&-0.41$ & N.A & \pm 0.104 \\
\hline Sarvgad-Moghaddam et al [37] & $1.52 \& 1.54$ & $1.41 \& 1.42$ & 1.38 & \pm 0.36 & \pm 0.21 & \pm 0.12 \\
\hline Meneghini et al [38] & 1.32 & 1.38 & 1.41 & \pm 0.40 & \pm 0.22 & \pm 0.10 \\
\hline Present Study & 1.52 & 1.58 & 1.56 & \pm 0.45 & \pm 0.21 & \pm 0.098 \\
\hline
\end{tabular}

previous studies, due to flow separation from the inner and outer sides of the cylinders, two vortical structures are developed downstream of the cylinders. In the stationary cases, it is expressible that at a small gap space between the cylinders, due to a reduction in the flow passage between the cylinders, a jet-like flow is developed between the cylinders. With an increase in the gap space between the cylinders, the effects of the jet-like flow attenuate and hence, the recirculating regions downstream of the cylinders become symmetric as that of the single cylinder in the free-stream flow. In the case of $\mathrm{RS}=1$, at $\mathrm{G} / \mathrm{D}=1.5$, while the inner vortex of the upper cylinder disappears and the outer vortex minimizes, downstream of the lower cylinder, both inner and outer vortices expand. A further increase of $\mathrm{RS}$ at $\mathrm{G} / \mathrm{D}=1.5$ collapses the inner and outer vortices of the lower cylinder gradually and, more or less, the fluid rotating zone dominates around the upper cylinder. In the case of $\mathrm{G} / \mathrm{D}=2.0$, although asymmetric flow patterns are seen downstream of the upper and lower cylinders at $\mathrm{RS}=1$, however, it seems among all gap spaces under consideration, the vortex strength of the lower cylinder is strong so that at $\mathrm{RS}=4$, a large scale vortical structure of the lower cylinder can be observed which shifts toward the outer side of the upper cylinder due to high rotating effect.

Table 3. Validation of the applied computer code against the available data for the single stationary cylinder.

\begin{tabular}{lc}
\hline Correlation and data & $\overline{N u}$ \\
\hline Hilpert and Forsch [39] & 15.43 \\
Churchill and Bernstein [40] & 16.63 \\
Harimi and Saghafian [41] & 16.39 \\
Present study & 17.65 \\
\hline
\end{tabular}

Finally, in the $\mathrm{G} / \mathrm{D}=3.0$, it is expressible that the interaction between the cylinders is low regardless of RS. As a result, in this subsection, it can be concluded that the sensitivity of the upper cylinder with respect to the rotation in the co-rotating scenario is considerably higher than that of the lower cylinder in which this sensitivity level of the upper cylinder decreases as a function of the G/D.

Figure 3 demonstrates the instantaneous vorticity fields at an arbitrary flow time for all cases under consideration. It should be said that the solid and dashed lines show the positive and negative voracities, respectively. In the stationary cases, at a small gap space such as $\mathrm{G} / \mathrm{D}=1.5$, due to development of a strong jet-like flow in the gap space, the pressure and shear stress distributions have considerable differences between the inner and outer sides of both upper and lower cylinders. In such cases, separation of the flow at the inner sides of the cylinders is expected to occur at higher angles as demonstrated by previous researchers [42]. Therefore, the shed vortices from the outer sides of the cylinders are larger than those of the inner sides with different shedding frequency. Increasing the gap space at the stationary case balances the pressure and shear stress distributions at the inner and outer sides due to a reduction in the jet-like effects so that, at $\mathrm{G} / \mathrm{D}=3.0$, the Karman vortex street can be seen like as a single stationary cylinder in the free-stream. At RS = 1, although the vortex shedding process occurs, however, the shed vortices from the cylinders are oriented toward the upper side due to the rotating effect. The suppression of the vortex shedding is seen clearly at $\mathrm{RS}=2$ regardless of the gap space between the cylinders as indicated in figure 3 . At $\mathrm{RS}=3$, the vortex shedding process is completely collapsed for $\mathrm{G} / \mathrm{D}=3.0$ but already occurred at the other G/D values. In the rotating speed of 4, while there is no vortex shedding phenomenon at 


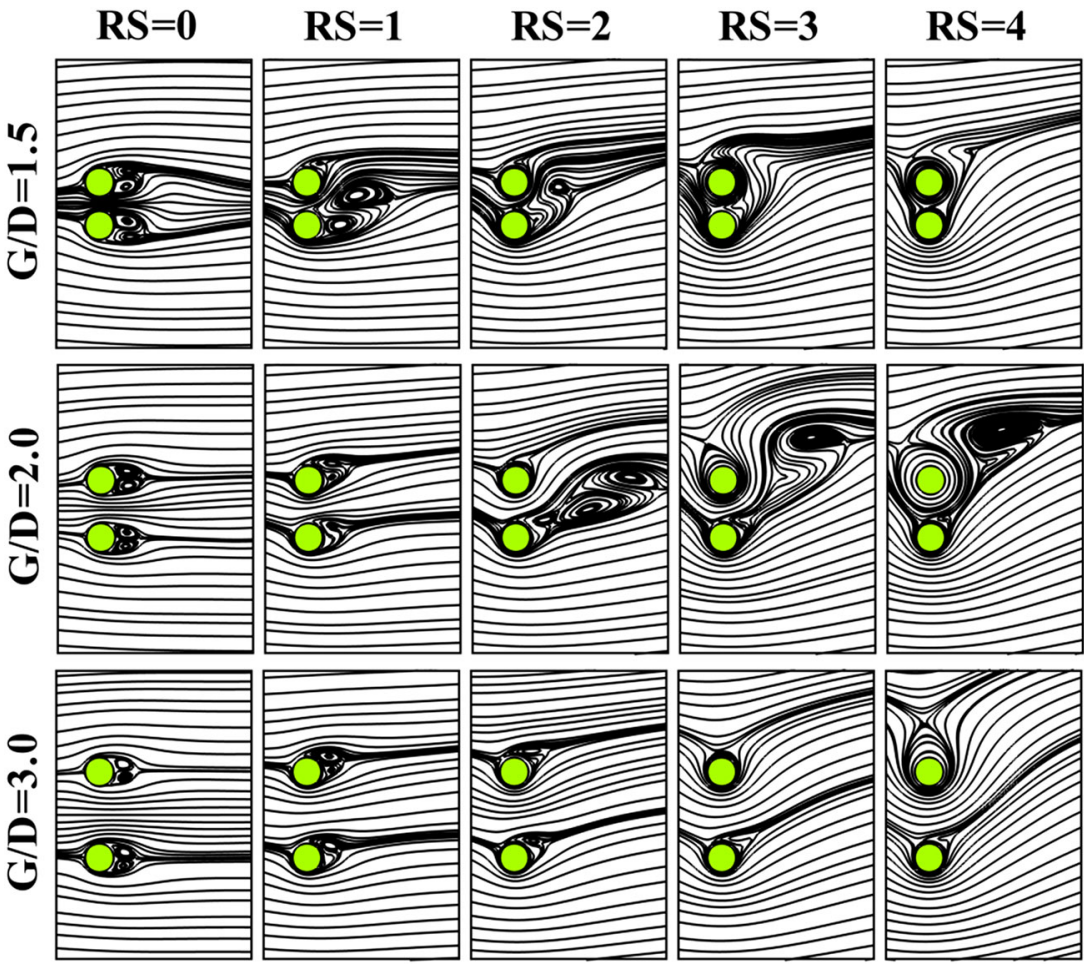

Figure 2. Time-averaged flow patterns at different G/D and RS values.
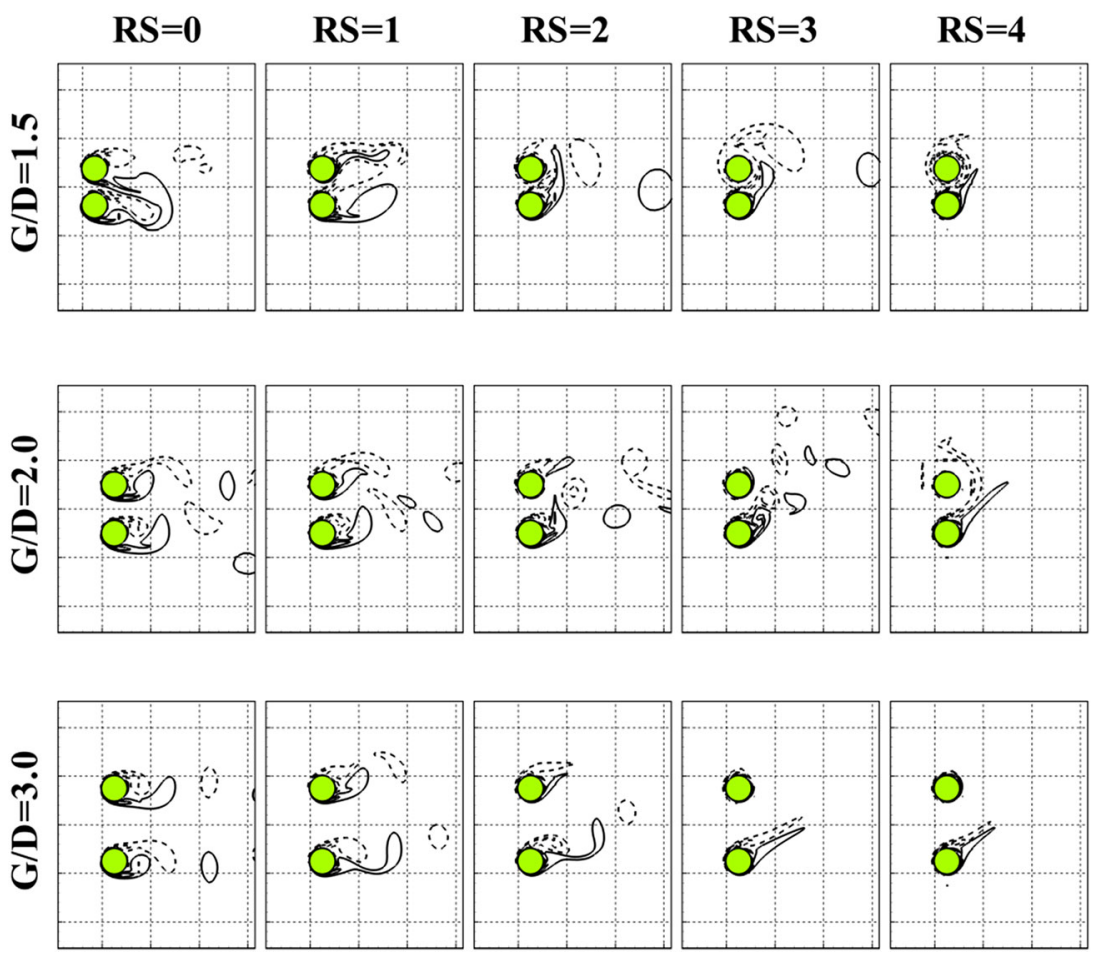

Figure 3. Instantaneous vorticity fields at an arbitrary time for all cased under consideration (contour level: $\omega_{\max }= \pm 2.85$ and $\Delta \omega=0.285)$.

$\mathrm{G} / \mathrm{D}=3.0$, at $\mathrm{G} / \mathrm{D}=1.5$ and 2.0 this occurrence minimizes and in this situation, it is not expected the existence of the large-scale vortices downstream of the cylinders.
Figure 4 shows the vortex shedding evolution at various RS values for $G / D=3.0$. It is clearly seen that in the case of $\mathrm{RS}=0$ and 1 , the vortex shedding process occurs for 


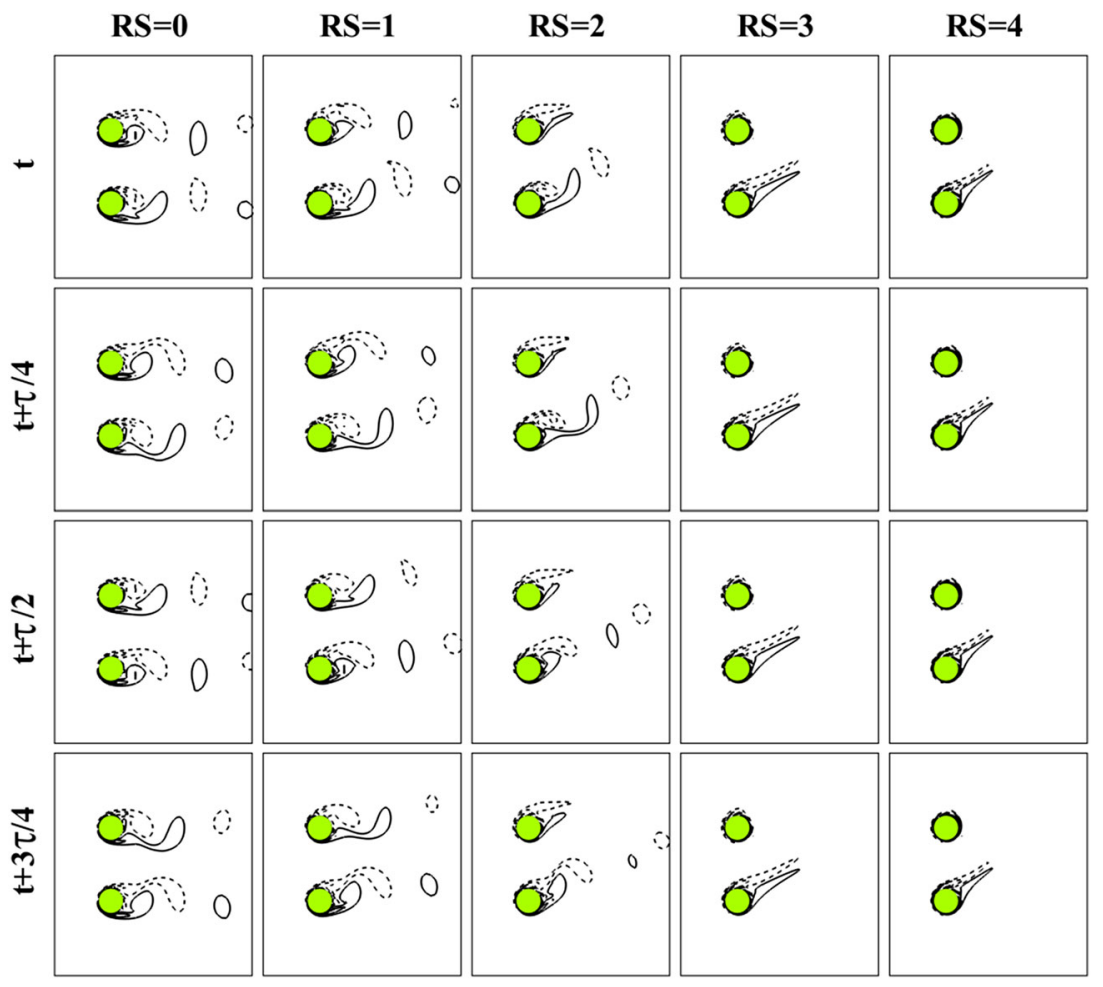

Figure 4. Vortex shedding evolution at various RS values for $\mathrm{G} / \mathrm{D}=3.0$ (contour level: $\omega_{\max }= \pm 3$ and $\Delta \omega=0.3$ ).
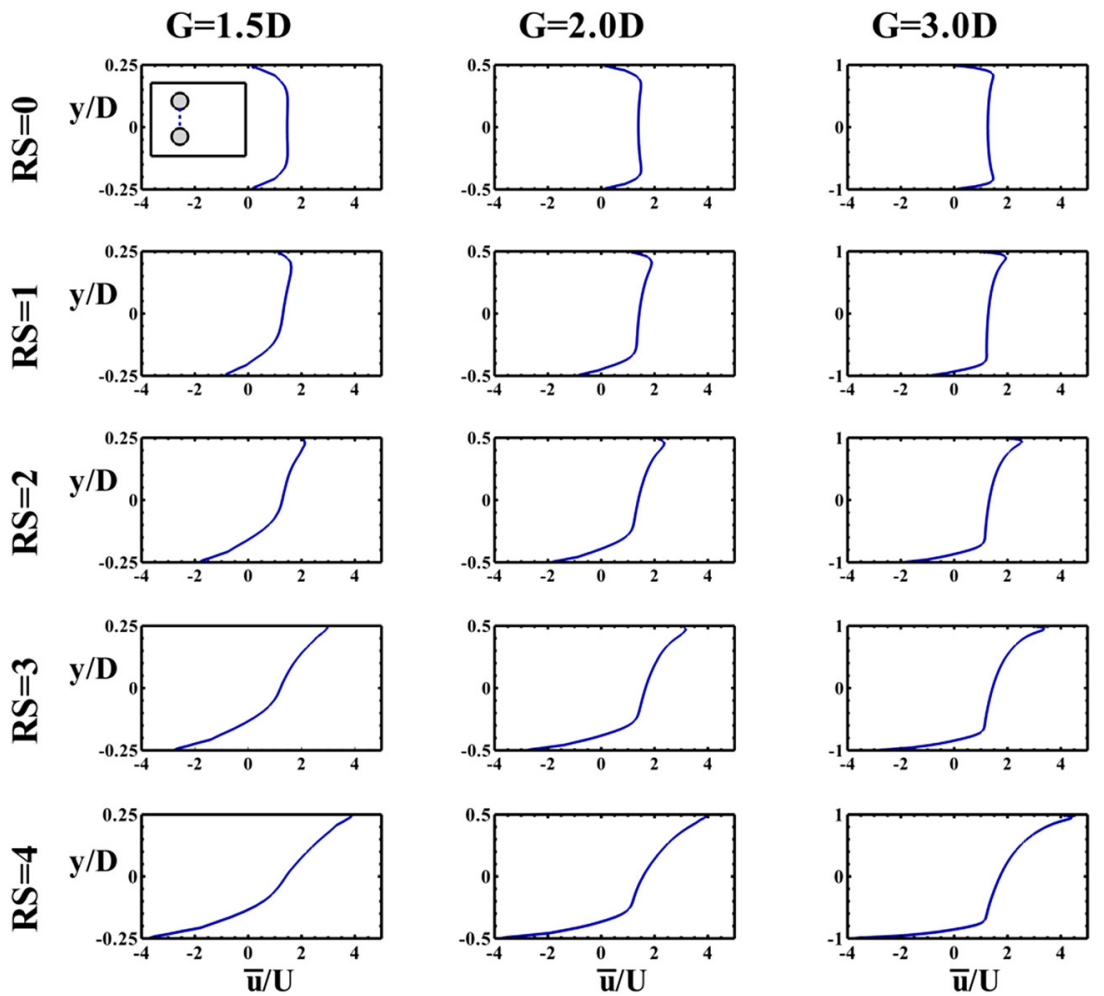

Figure 5. Distributions of the time-averaged streamwise velocity in the gap space between the cylinders at different G/D and RS values. 

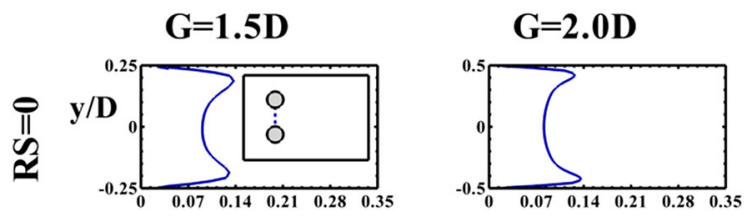

$\mathbf{G}=\mathbf{3 . 0 D}$
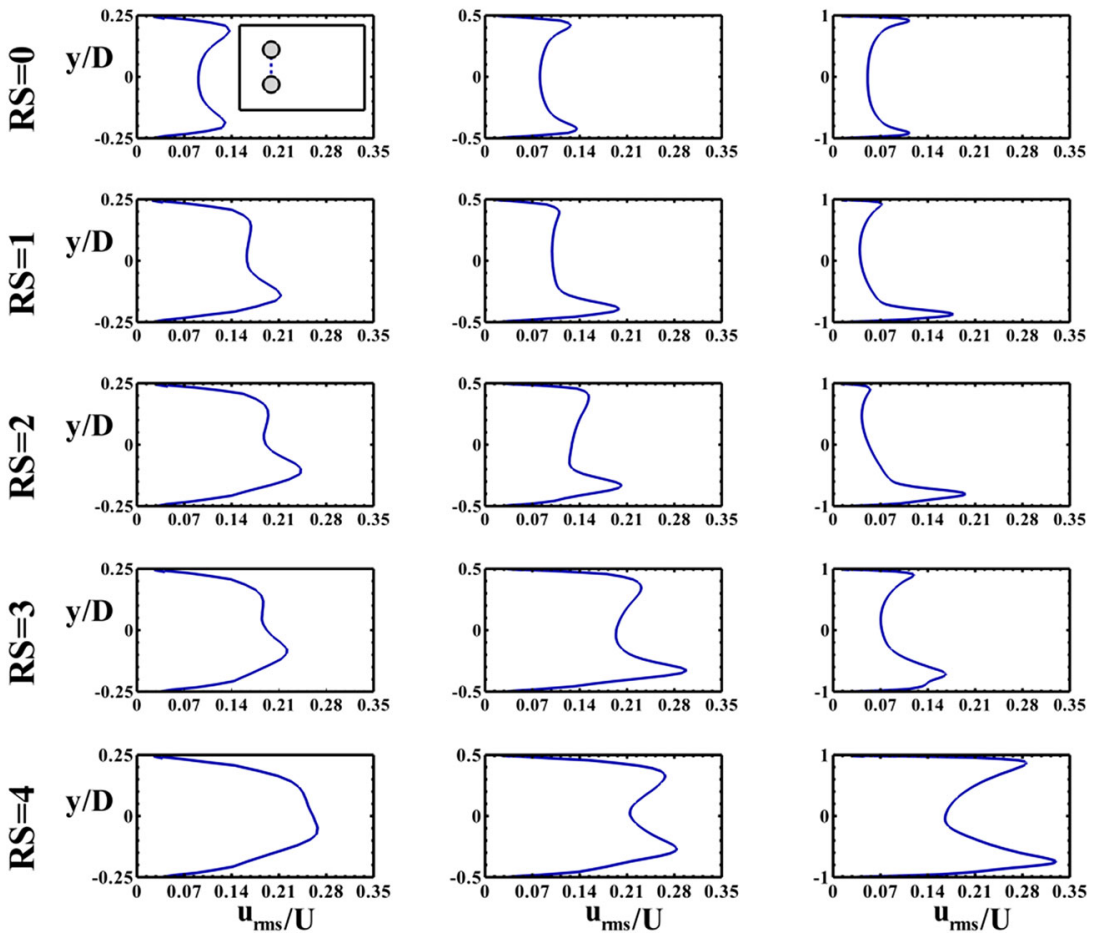

Figure 6. Distributions of root mean square (rms) of streamwise velocity in the gap space between the cylinders at different G/D and RS values.
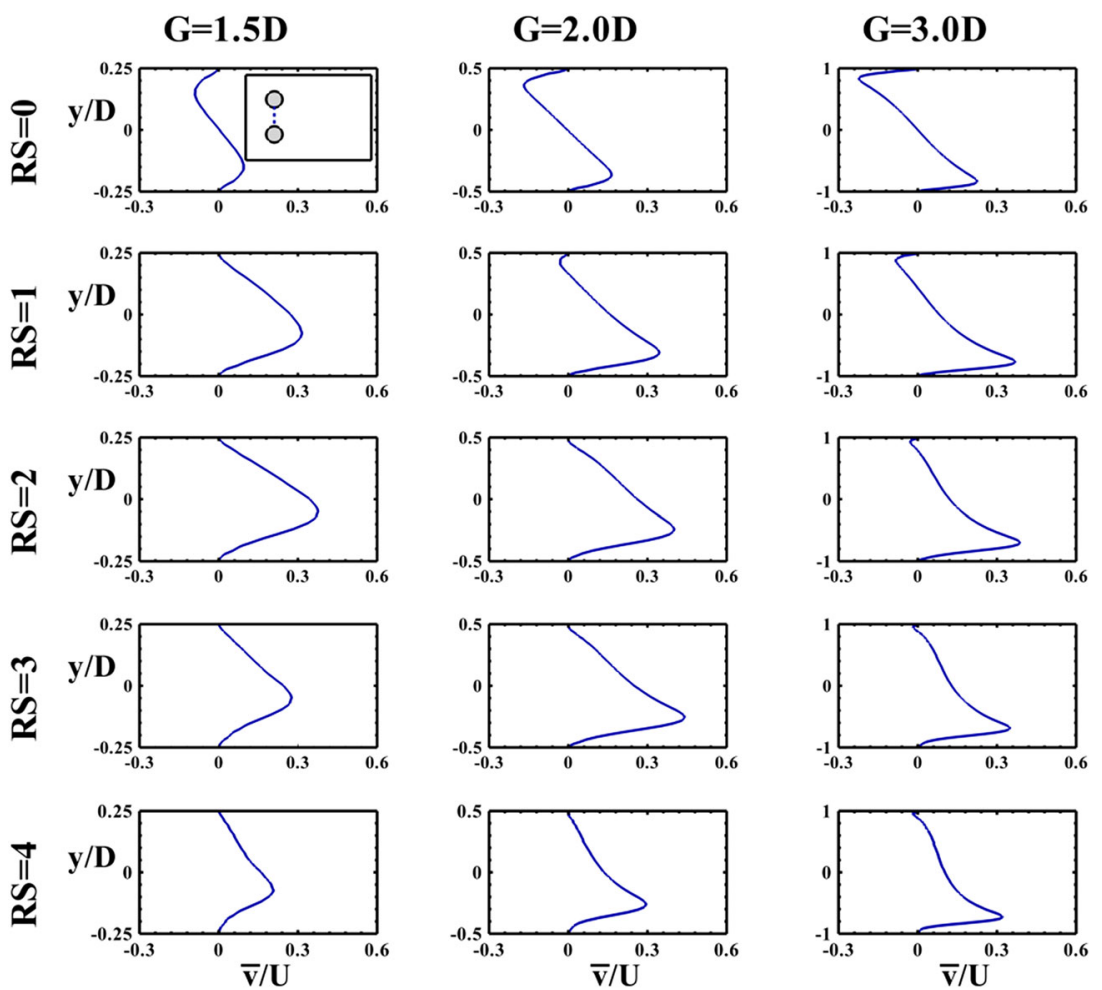

Figure 7. Distributions of the time-averaged vertical velocity in the gap space between the cylinders at different G/D and RS values. 

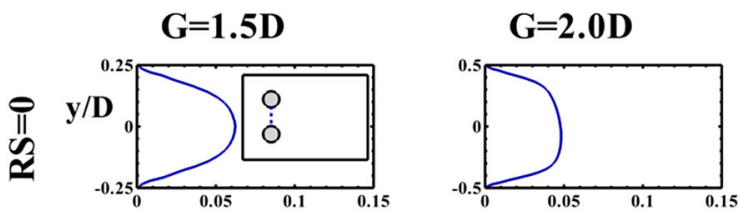

G=3.0D
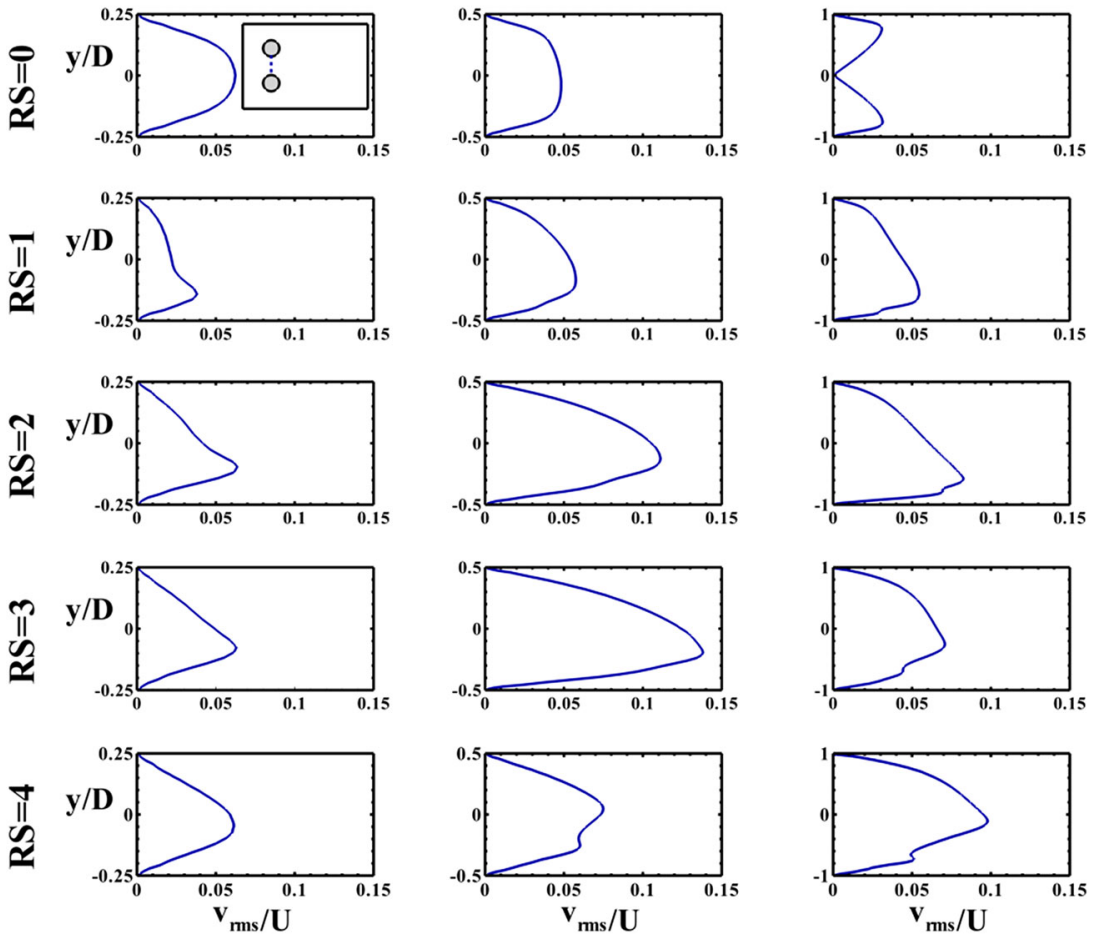

Figure 8. Distributions of root mean square (rms) of vertical velocity in the gap space between the cylinders at different G/D and RS values.
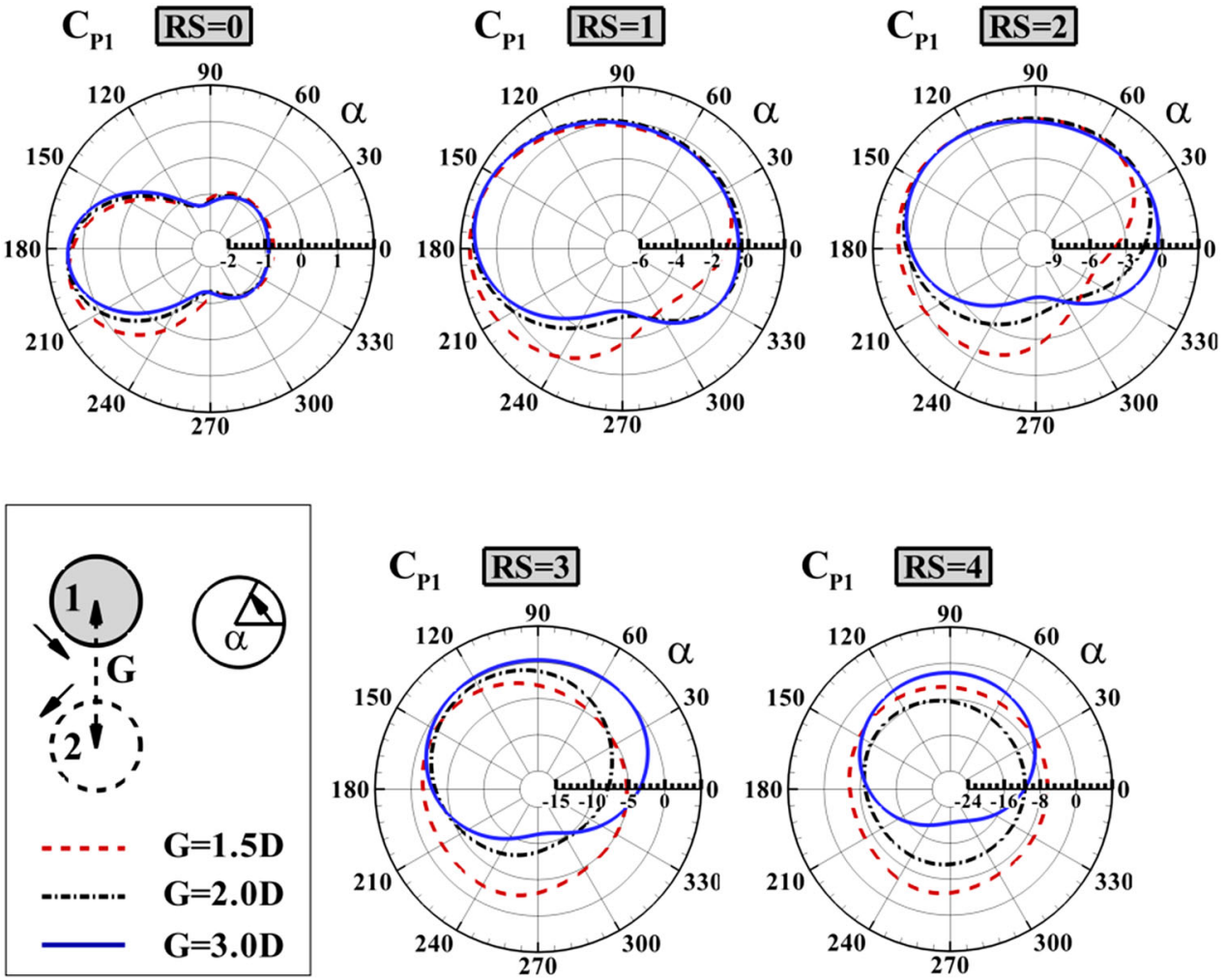

Figure 9. Distributions of the local pressure on the upper cylinder at different G/D and RS values. 

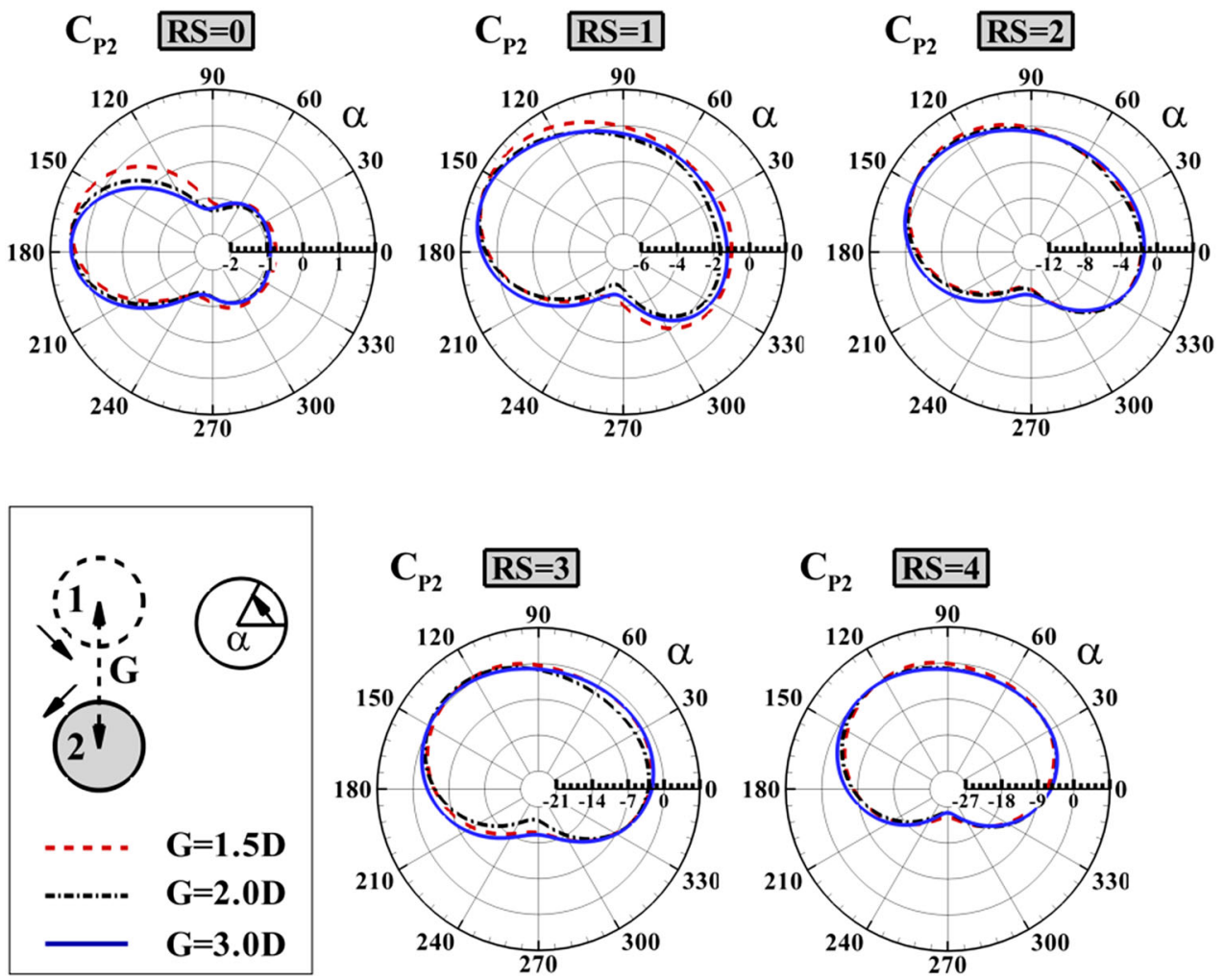

Figure 10. Distributions of the local pressure on the lower cylinder at different G/D and RS values.

both upper and lower cylinders. After RS $=1$, in RS $=2$, the vortex shedding process suppresses for the lower cylinders and collapses for the upper cylinder. This phenomenon finally disappears for both cylinder at $\mathrm{RS} \geq 3$.

Figure 5 illustrates distributions of the time-averaged stream-wise velocity at the gap space between two co-rotating cylinders at various G/D and RS values. It should be noted that the value of the stream-wise velocity is normalized with respect to the free-stream velocity. As expected, the time-averaged stream-wise velocity between two stationary side-by-side cylinders shows a symmetric distribution at the gap space. Due to the development of the jet-like flow between the cylinders, the velocity is high at the center of the gap space in the case of $\mathrm{G} / \mathrm{D}=1.5$. However, increasing the gap space between the stationary cylinders attenuates the jet-like effect at the gap space and hence, the maximum velocity occurred at this location reduces as a function of the G/D. Co-rotating the side-byside cylinders provides the asymmetric stream-wise velocity distributions at the gap space as seen in figure 5 . At the low RS value such as RS $=1$, the values of the stream-wise velocity amplify near the upper cylinders while is attenuating near the lower cylinder since the flow orientates toward the upper side due to rotation effect. Regardless of the G/D, the stream-wise velocity distribution at the gap space shows a gradual increase from the lower cylinder toward the upper one. A further increase in RS value augments the velocity gradient between the lower and upper cylinders.

In order to understand the effects of the gap space between two co-rotating side-by-side cylinders on the flow behavior, distributions of the root mean square of streamwise velocity normalized by free-stream velocity are demonstrated in figure 6 at the gap space between the cylinders. As seen, in stationary cases, two maximum points can be observable close to the cylinders. These peak points are mainly due to the vortex shedding process. By rotating the cylinders both in the counterclockwise direction, the rate of the unsteadiness grows considerably near the lower cylinder while attenuates near the upper cylinder as an inverse function of G/D. This occurrence is due to the high interaction between the free-stream flow and rotating flow developed around the lower cylinder in comparison to the upper cylinder. Further increasing RS amplifies the stream-wise velocity fluctuations in the gap space so that by dominating the fluid rotating zone around the cylinders at $\mathrm{RS}=4$, the maximum rate of the unsteadiness can be reported near the center of the gap at $\mathrm{G} / \mathrm{D}=1.5$ and close to the cylinders at $\mathrm{G} / \mathrm{D}=2.0$ and 3.0.

Figure 7 presents time-averaged vertical velocity distributions at the gap space between two co-rotating sideby-side cylinders at different G/D and RS values. Similar to the reported data in figures 5 and 6 , the values of vertical velocities are also normalized with respect to the 

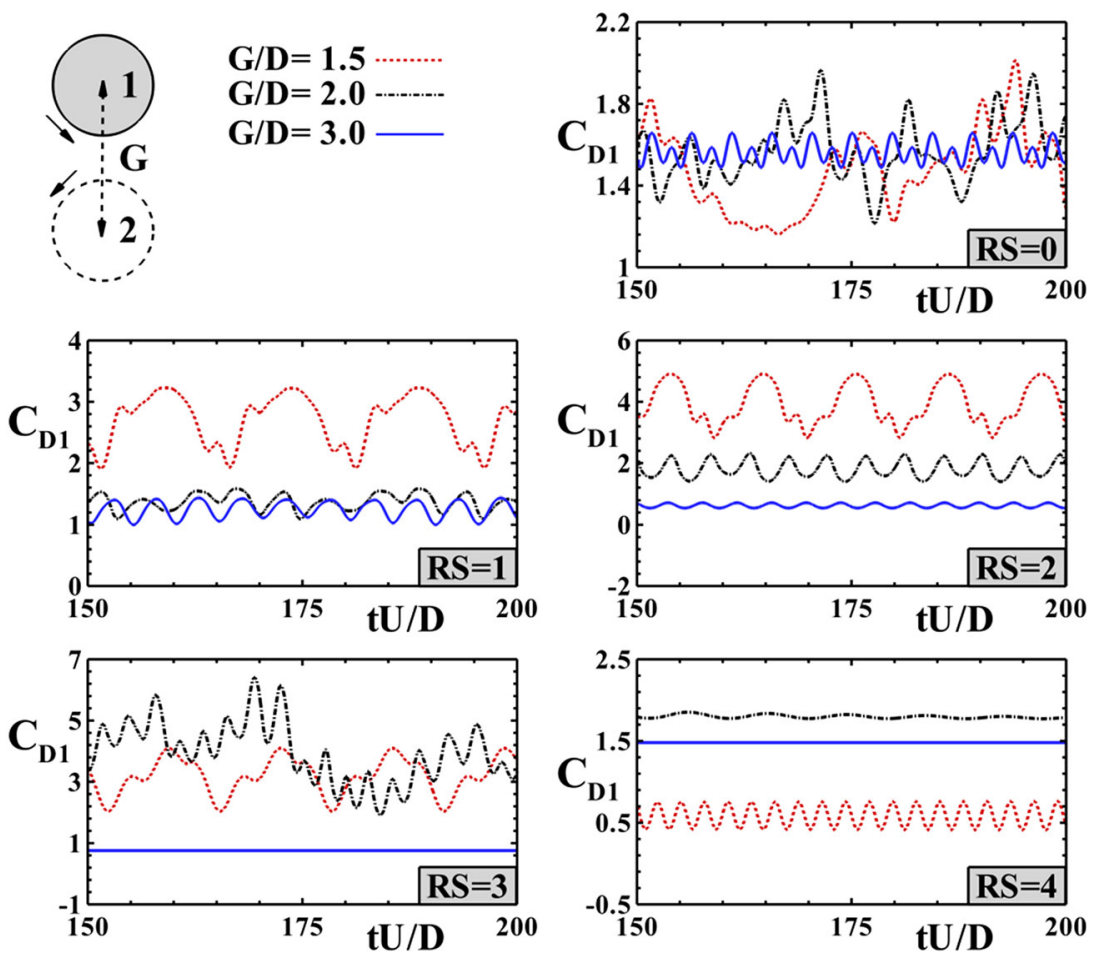

Figure 11. Time-histories of the drag coefficient of the upper cylinder at different G/D and RS values.
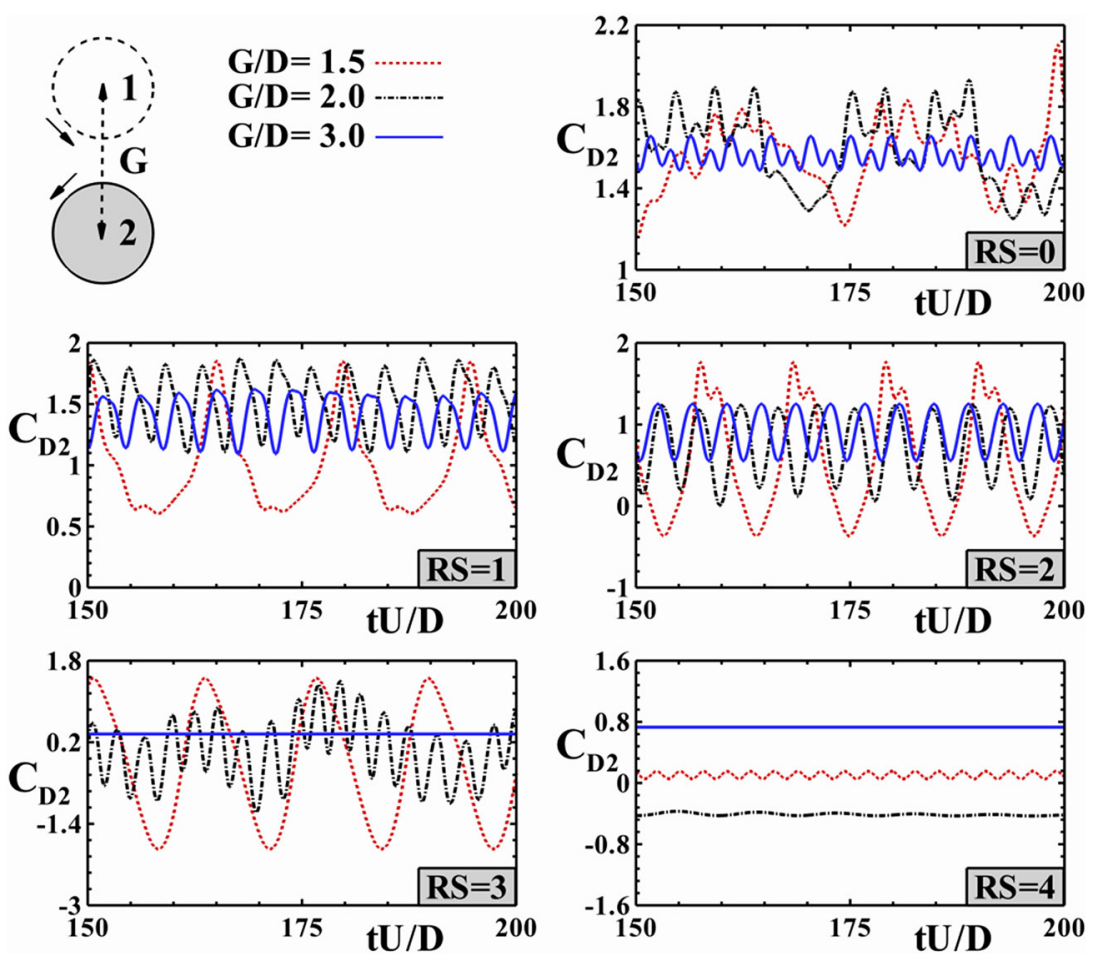

Figure 12. Time-histories of the drag coefficient of the lower cylinder at different G/D and RS values.

free-stream velocity. In the case of the stationary side-byside cylinders, regardless of the G/D value, a symmetric distribution with two negative and positive peaks is seen.
Development of the vertical velocity at the gap between the cylinders is due to an interaction between the free-stream flow and cylinders. Increasing the G/D enhances the 
vertical velocity magnitudes between the cylinders at the stationary scenario. Co-rotating the cylinders enhances the vertical velocity magnitudes near the lower cylinder and collapses their magnitudes near the upper one as a function of G/D and RS. However, as seen, in the case of RS = 4 in which the fluid rotating zones are dominated around both cylinders, a gradual reduction in the vertical velocity is seen between the cylinders for all G/D values.

Figure 8 demonstrates the root mean square distributions of the vertical velocity at the gap space between two corotating side-by-side cylinders at different $\mathrm{G} / \mathrm{D}$ and RS values. In the stationary case $(\mathrm{RS}=0)$, the maximum vertical velocity fluctuations occur at the center of the gap space in $\mathrm{G} / \mathrm{D}=1.5$ and 2.0. However, at large gap space such as $\mathrm{G} / \mathrm{D}=3.0$, two peak point are developed near the upper and lower cylinders. Co-rotating the cylinders shifts the vertical velocity fluctuations toward the lower cylinder up to $\mathrm{RS}=3$. In the range of $1 \leq \mathrm{RS} \leq 3$, the maximum vertical velocity unsteadiness develops at $\mathrm{G} / \mathrm{D}=2$. At high

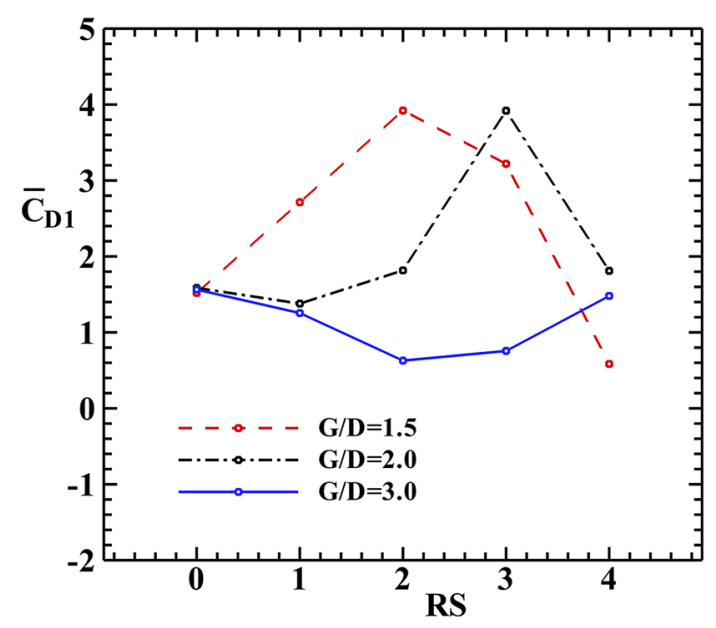

(a)

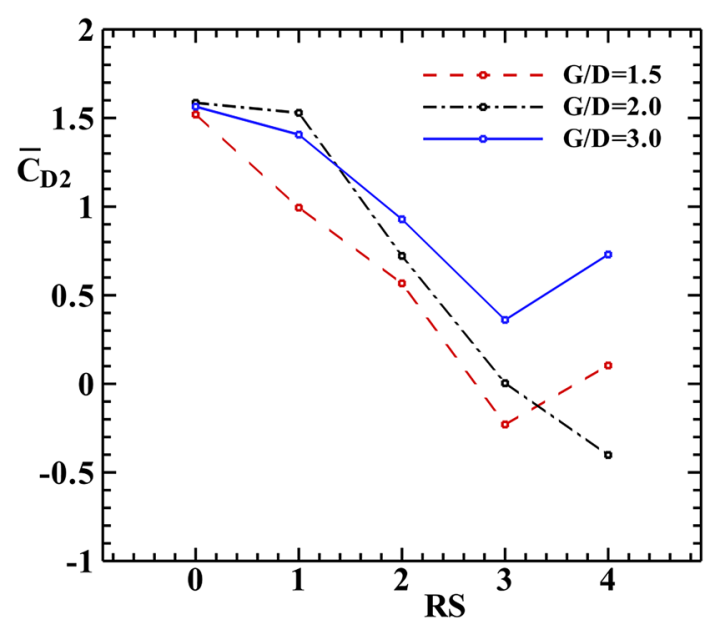

(b)

Figure 13. Variation of the mean drag coefficient with G/D and RS; (a) upper cylinder, (b) lower cylinder.
RS value of 4 , it seems the peak point moves toward the center of the gap space at all G/D values and in this RS value, the vertical velocity fluctuations grow as a function of $\mathrm{G} / \mathrm{D}$.

One of the main challenges in the co-rotating side-byside cylinders is the distribution of the local pressure on each upper and lower cylinders. In this regard, figures 9 and 10 illustrate the local pressure distributions at various $G / D$ and RS values on the upper and lower cylinders, respectively. In the case of the upper cylinder, as demonstrated in figure 9 , maximum pressure at $\mathrm{RS}=0$ is developed at the front stagnation point regardless of the G/D value. In this stationary case, the interaction between the upper and lower cylinders provides an asymmetric pressure distribution for small and moderate G/D value. In other words, the pressure distribution of the upper stationary cylinder is different between the half-outer and half-inner sides which forms the lift force as an inverse function of G/D. However, at high $\mathrm{G} / \mathrm{D}$ value such as 3.0, it seems the interaction between the stationary side-by-side cylinders minimizes as seen in the first image of figure 9. The first occurrence, once the cylinders rotate, is shifting the front stagnation point in the clockwise direction for the upper cylinder. This displacement in the front stagnation point occurs as a function of G/D and RS. The second occurrence in this concept is the development of a non-symmetric pressure distribution between the half-outer and half-inner sides of the upper cylinder for each $\mathrm{G} / \mathrm{D}$ value providing a vertical force on the upper cylinder as a function of RS. The last occurrence in the rotating cases is domination of the negative pressure all over the upper cylinder in which the magnitudes of these negative pressures amplify with an increase in RS at each G/D. A noticeable result in this issue is the development of nearly symmetric pressure distribution at high RS and small G/D such as 1.5 which shows that at this RS and G/D values, all points on the upper cylinder experience a uniform pressure magnitude. Therefore, it is predictable that the magnitudes of the lift and drag coefficient at RS $=4$ and $\mathrm{G} / \mathrm{D}=1.5$ attenuate strongly. For the lower cylinder, in the case of the stationary cylinder, similar results of the upper cylinder can be reported for the lower cylinder. However, in the case of the rotating cylinders, it seems the effect of the gap space between the side-by-side cylinders attenuates considerably for the lower cylinder. In other words, in comparison to the upper cylinder, gap space between the cylinders has a minor role on the pressure distribution over the lower cylinder regardless of the RS. Like as the upper cylinder, front stagnation point of the lower cylinder shifts in the clockwise direction as a function of RS. On the other hand, negative pressure magnitudes are seen all over the lower cylinder in which the magnitudes of these negative pressures amplify as a function of the RS.

Figures 11 and 12 illustrate time-histories of the drag coefficient under various G/D and RS values along the nondimensional time for upper and lower cylinders, 

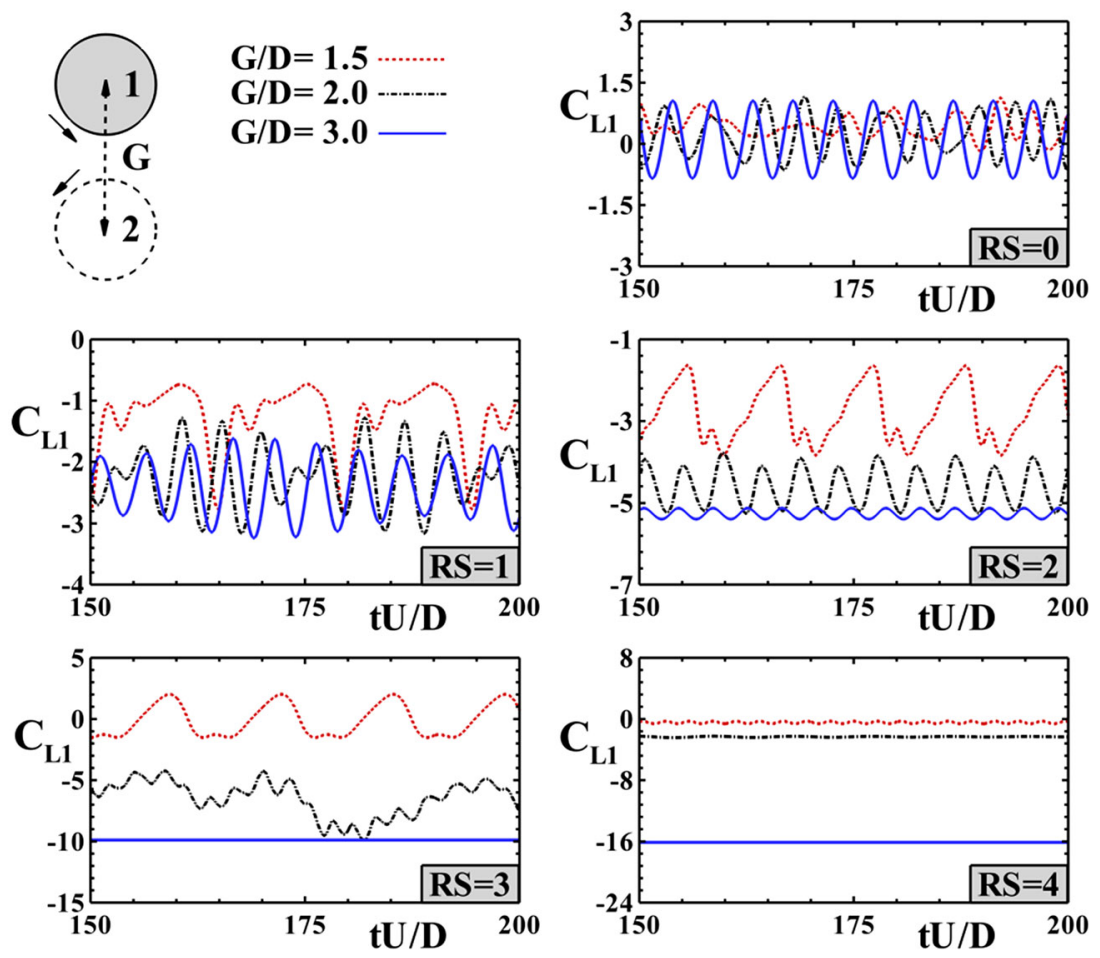

Figure 14. Time-histories of the lift coefficient of the upper cylinder at different G/D and RS values.
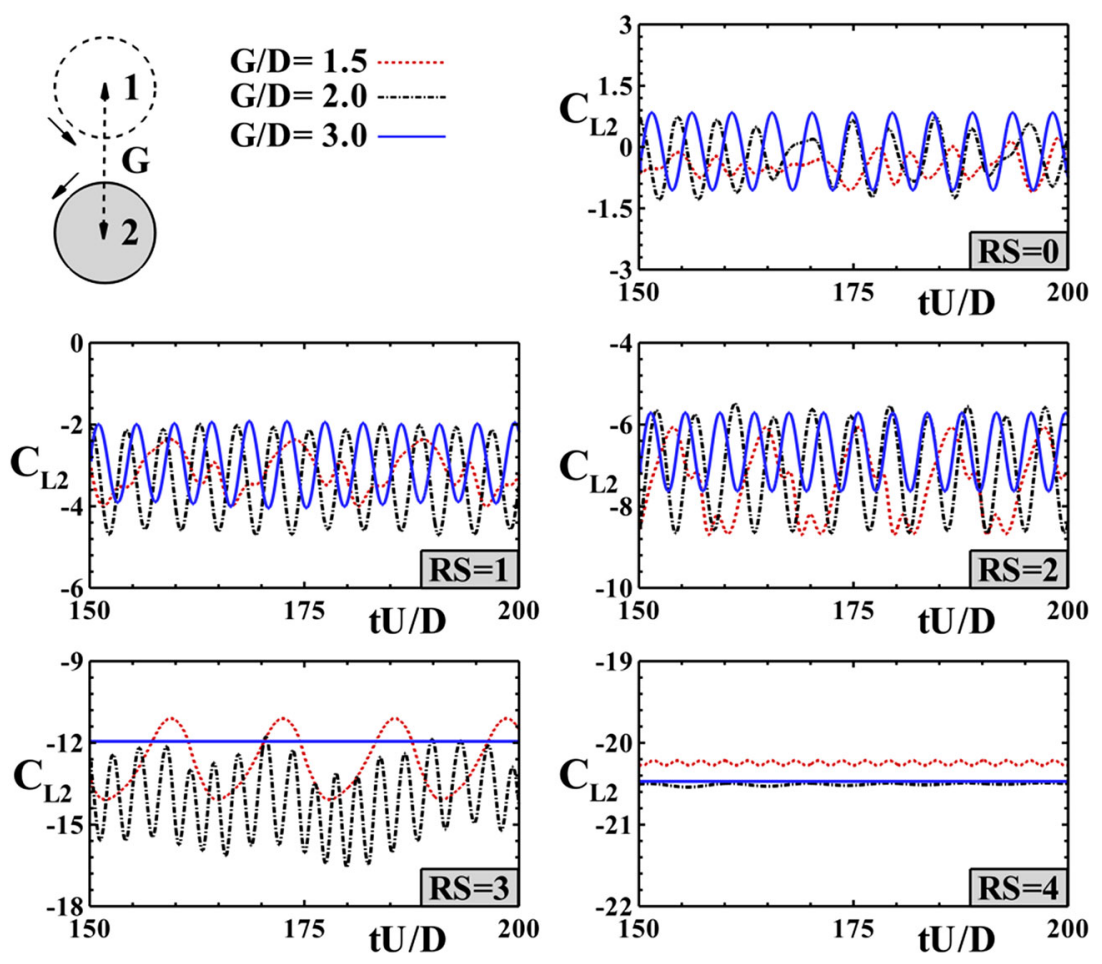

Figure 15. Time-histories of the lift coefficient of the lower cylinder at different G/D and RS values.

respectively. In the stationary case, for both upper and lower cylinders, time-histories of the drag coefficient are oscillating at all G/D values. These oscillations are due to vortex shedding from the sides of the cylinders. However, regardless of the $\mathrm{G} / \mathrm{D}$, the oscillations are irregular because of the different pressure distributions at the half-outer and 


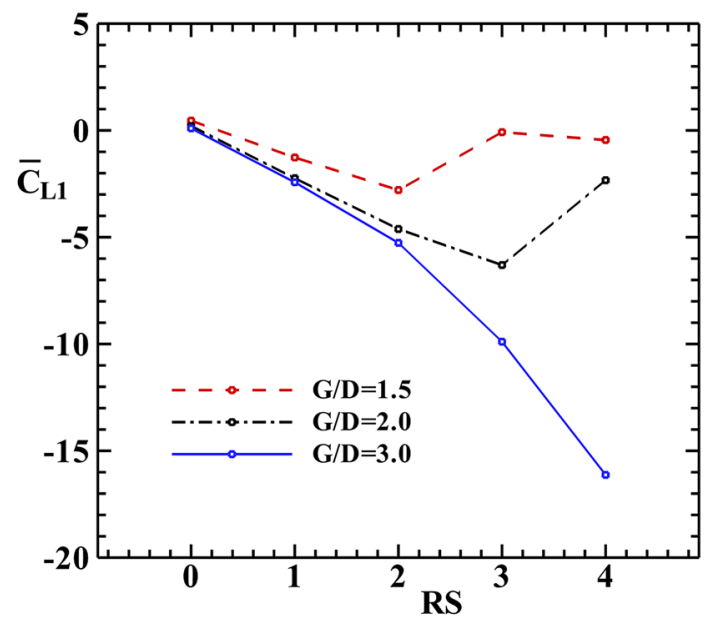

(a)

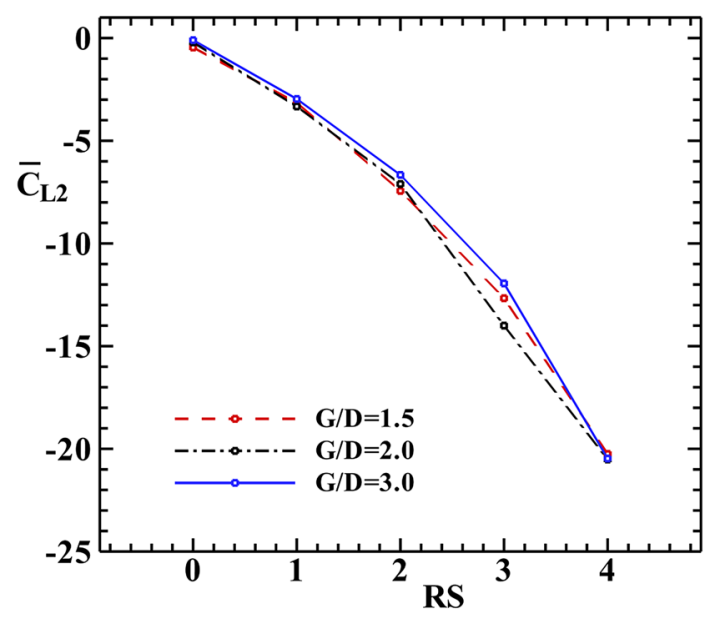

(b)

Figure 16. Variation of the mean lift coefficient with G/D and RS; (a) upper cylinder, (b) lower cylinder.

half-inner sides of both cylinders. By rotating the cylinders, it is observable that the vortex shedding process occurs up to $\mathrm{RS}=3$. However, the shedding frequency reduces as a function of RS. It is also expressible that this reduction in the shedding frequency is more evident for small and moderate G/D values than that of the large G/D case. An exception is reportable that at $\mathrm{RS}=1$, the shedding frequency and amplitude enhance in $\mathrm{RS}=1$ at $\mathrm{G} / \mathrm{D}=2.0$ and beyond this $\mathrm{RS}$ value, a systematic reduction in the flow unsteadiness can be observed. At RS $=3$, while the oscillations of drag coefficients disappear at $\mathrm{G} / \mathrm{D}=3.0$, these oscillations are seen at $\mathrm{G} / \mathrm{D}=1.5$ and 2.0. Finally, at $\mathrm{RS}=4$, as seen, the oscillations collapse considerably for small and moderate G/D values demonstrating the domination of a strong fluid rotating zone on the upper and lower cylinders.

In order to understand the effects of $\mathrm{G} / \mathrm{D}$ and $\mathrm{RS}$ values on the mean drag coefficient of two co-rotating cylinders, figures 13 (a) and (b) are provided. In the small $G / D=1.5$, the mean drag coefficient of the upper cylinder shows a rising variation with $\mathrm{RS}$ up to $\mathrm{RS}=2$ since the recirculating region of the lower cylinder moves toward the rear side of the upper cylinder as demonstrated in figure 2. Beyond the $\mathrm{RS}=2$, the mean drag coefficient falls with an increase in RS since the vortical structure of the upper and lower cylinders disappears and collapses, respectively. At the moderate $\mathrm{G} / \mathrm{D}=2.0$, despite the $\mathrm{G} / \mathrm{D}=1.5$, the mean drag coefficient reduces in the range $0 \leq \mathrm{RS} \leq 1$, increases at $1 \leq \mathrm{RS} \leq 3$, and falls beyond $\mathrm{RS}=3$. At the large $\mathrm{G} / \mathrm{D}=3.0$, the mean drag coefficient of the upper cylinder shows a descending variation with $\mathrm{RS}$ up to $\mathrm{RS}=2$ and beyond $\mathrm{RS}=2$, the drag coefficient increases gradually as a function of RS as illustrated in figure 13(a). Regarding the lower cylinder, at small and large G/D values, the mean drag coefficient shows a descending variation with RS up to $\mathrm{RS}=3$ due to an orientation of the recirculation region toward the upper side resulted by rotation of the lower cylinder and beyond this RS, the drag coefficient increases. However, the drag coefficient of the lower cylinder in the case of $\mathrm{G} / \mathrm{D}=2.0$ reduces as a function of $\mathrm{RS}$ at whole under consideration $\mathrm{RS}$ range. It should be mentioned that the drag coefficient for stationary side-by-side cylinders is in good agreement with previous studies.

Figures 14 and 15 show time-histories of the lift coefficient under various G/D and RS values along the non-dimensional time for upper and lower cylinders, respectively. Similar to the drag history, due to the vortex shedding process, temporal variations of the lift coefficients for stationary side-by-side cylinders are oscillating regardless of the G/D value. However, since the pressure distributions at the half-outer and half-inner sides of each stationary cylinder are different, hence, the oscillations are irregular and with an increase in the $\mathrm{G} / \mathrm{D}$ value up to $\mathrm{G} / \mathrm{D}=3.0$, it seems the oscillations become regular. For the upper rotating cylinder, the amplitudes of the lift force oscillations maximize at $\mathrm{RS}=1$ and beyond this critical value, the lift coefficient fluctuations attenuate as a function of RS. At $\mathrm{RS}=3$, the oscillation of the lift force for upper cylinder collapses at $\mathrm{G} / \mathrm{D}=3.0$ and minimizes at $\mathrm{RS}=4$ for $\mathrm{G} / \mathrm{D}=1.5$ and 2.0. Similar reports are expressible regarding the lower rotating cylinder.

Figures 16(a) and (b) present the mean lift coefficients as a function of G/D and RS for upper and lower side-by-side cylinders, respectively. Regarding the upper cylinder, at $\mathrm{G} / \mathrm{D}=3.0$, the negative lift coefficient enhances as a function of RS. However, at $\mathrm{G} / \mathrm{D}=1.5$ and 2.0, the lift coefficients reduce after $\mathrm{RS}=2$ and 3 , respectively. This occurrence is mainly due to a reduction in the pressure difference between the half-outer and half-inner sides of the upper cylinder at $\mathrm{G} / \mathrm{D}=1.5$ and 2.0 at high $\mathrm{RS}$ values despite the $\mathrm{G} / \mathrm{D}=3.0$ as discussed in figure 9 . In the case of the lower cylinder, it is observable that, regardless of the G/D, the negative mean lift coefficients amplify with an increase in RS. The maximum difference among the lift coefficients of $\mathrm{G} / \mathrm{D}=1.5,2.0$, and 3.0 occurs in $\mathrm{RS}=3$. 

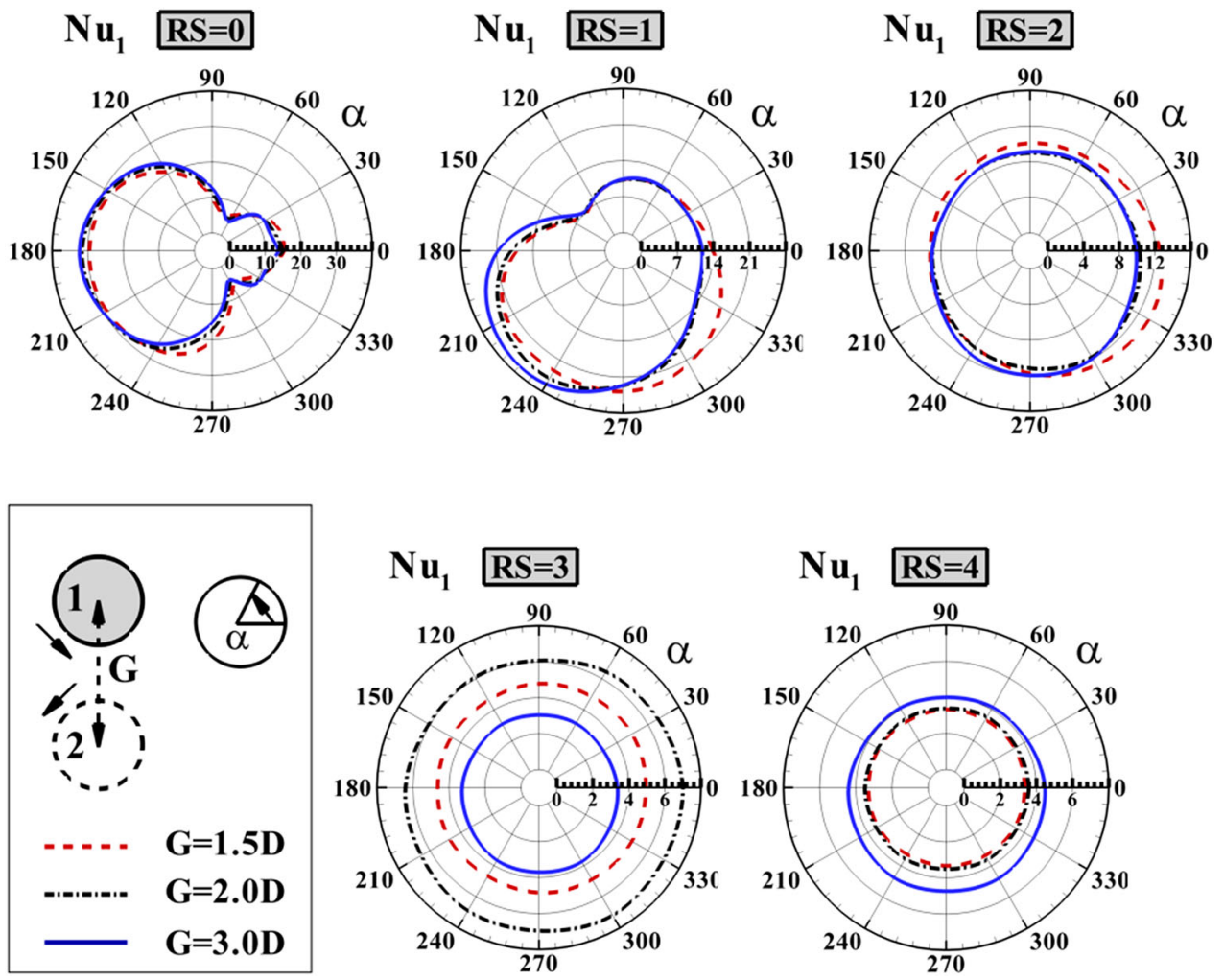

Figure 17. Distributions of the local Nusselt number on the upper cylinder at different G/D and RS values.
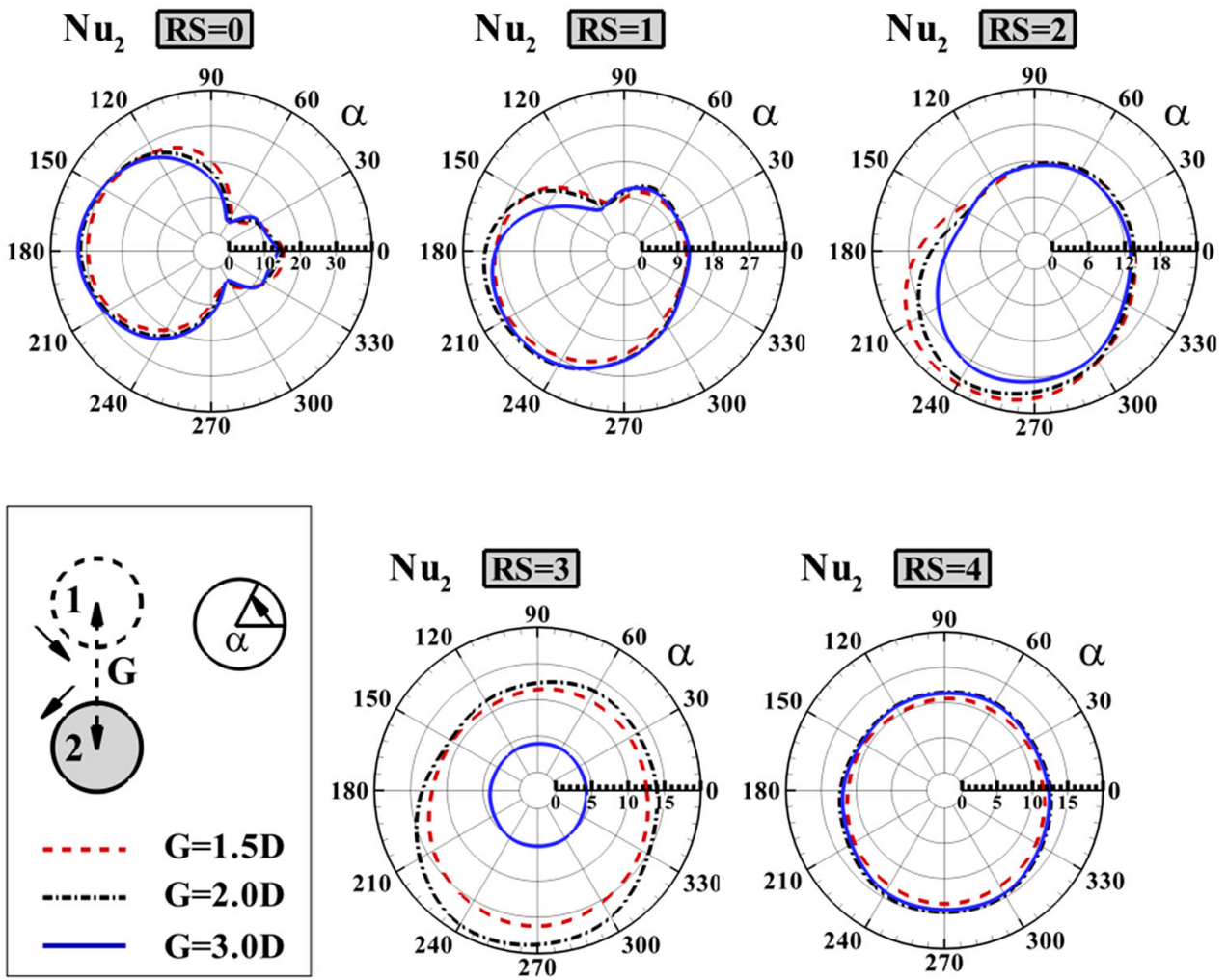

Figure 18. Distributions of the local Nusselt number on the lower cylinder at different G/D and RS values. 


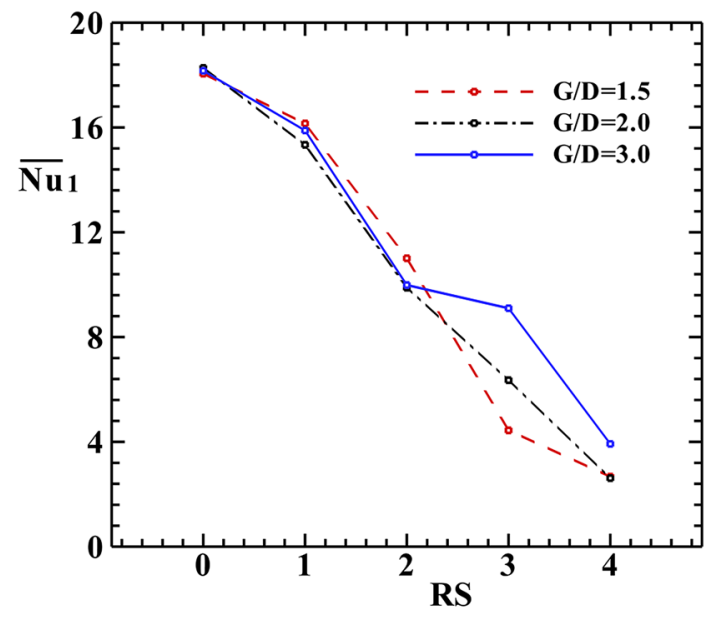

(a)

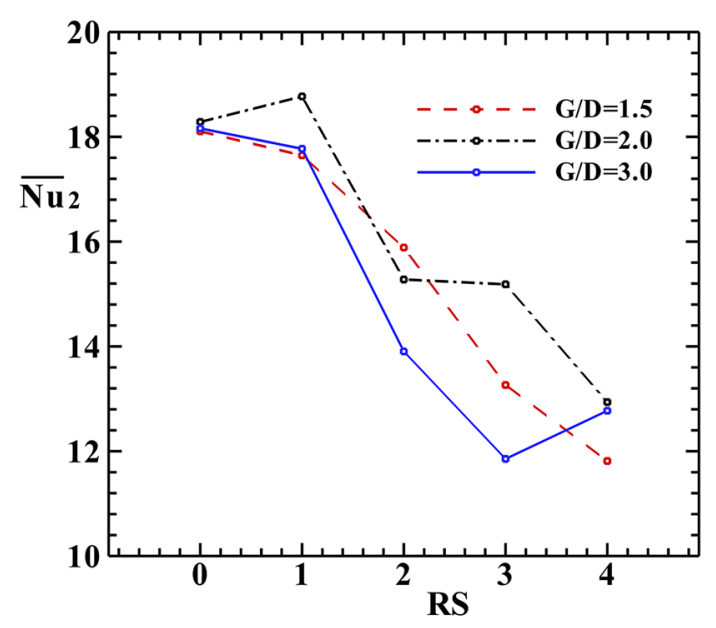

(b)

Figure 19. Variation of the mean Nusselt number with G/D and RS; (a) upper cylinder, (b) lower cylinder.

Development of the negative lift coefficient on the lower rotating cylinder in whole G/D and RS values indicates that the lower cylinder shows a tendency to move toward the negative vertical direction.

Figures 17 and 18 show the local Nusselt number distributions as a function of the G/D and RS for upper and lower cylinders, respectively. In accordance with previous studies, for both upper and lower stationary cylinders, the rate of the heat transfer is maximum at the front stagnation point and with getting away from this location in the angular direction, the rate of the heat transfer attenuates gradually due to growing of the boundary layer thickness so that the minimum heat exchange rates are seen at $\alpha=60^{\circ}$ and $300^{\circ}$. After these two locations, the heat transfer rate enhances due to a higher rate of the unsteadiness downstream of the cylinders resulted by flow separation from the outer and inner sides of each cylinder. Co-rotating the cylinders in counterclockwise direction enhances the pressure on the half-outer side of the upper cylinder and

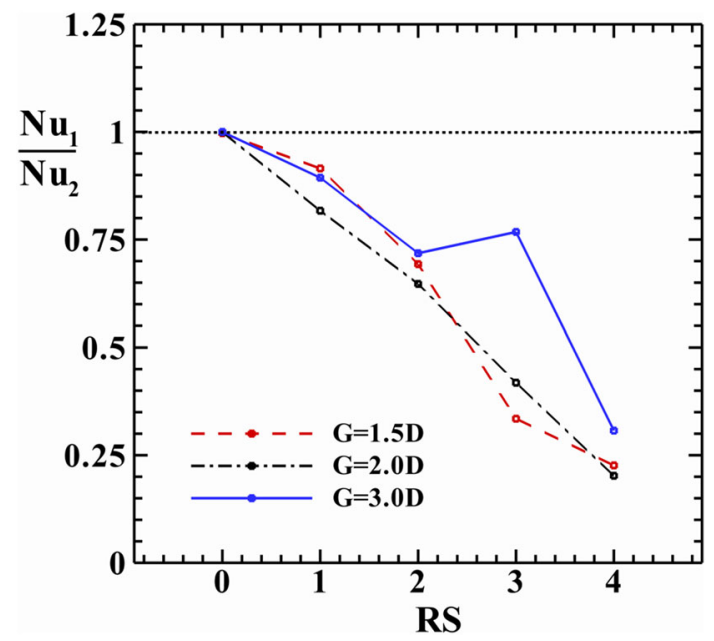

Figure 20. Variation of the relative mean Nusselt number with G/D and RS.

half-inner side of the lower cylinder and hence, the local Reynolds number at these location reduces considerably and reduction of the heat transfer rate is expected accordingly. Despite this, at the half-inner side of the upper cylinder and half-outer side of the lower cylinder, the local Reynolds number augments and the rate of the heat exchange enhances. At a higher rotating speed such as RS $=2$, the rate of the heat transfer difference between the half-outer and half-inner sides of the upper cylinder attenuates and after that, it seems a nearly uniform Nusselt number distributions develop on the upper cylinder at all G/D values. This occurrence is mainly due to dominating the fluid rotating zone around the cylinder and in this zone, only conductive heat transfer occurs. However, for the lower cylinder, it is clearly seen that the non-uniform Nusselt number distributions occur up to $\mathrm{RS}=3$ and at $\mathrm{RS}=4$, a nearly uniform heat transfer rate occurs for all $\mathrm{G} / \mathrm{D}$ values.

Although consideration of the local Nusselt number on two co-rotating cylinders is important in terms of the surface temperature and heat flux control of two co-rotating side-by-side cylinders, however, having the mean Nusselt number is also important from the engineering viewpoint. Figures 19(a) and (b) show the variations of the mean Nusselt number against the G/D and RS values for upper and lower cylinders, respectively. As seen, for the upper cylinder, the effect of the gap space between two co-rotating side-by-side cylinders becomes more evident at $R S \geq 2$ in which the value of the Nusselt number enhances as a function of G/D. However, at all G/D values, with an increase in RS, the Nusselt number decreases. In the case of the lower cylinder, variation of the Nusselt numbers shows a complex behavior. That is, at the small $\mathrm{G} / \mathrm{D}=1.5$, similar to the upper cylinder, the distribution of the Nusselt number shows a descending variation with RS. At the moderate $\mathrm{G} / \mathrm{D}=2.0$, the maximum rate of the heat transfer occurs at $\mathrm{RS}=1$, and at the large $\mathrm{G} / \mathrm{D}=3.0$, the heat transfer 
between the rotating cylinder and the fluid flow reduces up to $\mathrm{RS}=3$ and beyond this $\mathrm{RS}$ value, the heat exchange rate enhances gradually. In other words, it is expressible that the effect of the G/D on the heat transfer of the lower cylinder is more obvious than the upper cylinder.

For enhanced visualization, figure 20 compares the relative Nusselt number (the ratio of the Nusselt number for the upper cylinder per that of the lower cylinder) at various $\mathrm{RS}$ and G/D values. It is observable that this ratio is less than one at whole RS and G/D values demonstrating the lower heat transfer rate of the upper cylinder in comparison to that of the lower cylinder. For all G/D values, the relative Nusselt number reduces with an increase in RS except for $\mathrm{G} / \mathrm{D}=3.0$ at which the relative heat transfer partially enhances at $\mathrm{RS}=3$.

\section{Conclusion}

In this numerical study, heat and fluid flow around two corotating side-by-side cylinders has been investigated at constant Reynolds and Prandtl numbers of 200 and 7.0, respectively. In this regard, several non-dimensional gap spaces such as $\mathrm{G} / \mathrm{D}=1.5,2.0$, and 3.0 and also various rotating speeds in the range of $0 \leq \mathrm{RS} \leq 4$ were considered. Several important results have been obtained in the present study which can be summarized as follows.

- Co-rotating two side-by-side cylinders deforms the wake region downstream of both cylinders as a function of RS. The vortex strength of the lower cylinder is realized to be stronger in comparison to that of the upper cylinder at all G/D values.

- In the gap space between the cylinders, it was revealed that, regardless of the G/D and RS values, the maximum stream-wise and vertical velocity magnitudes were developed near the upper and lower cylinders, respectively. However, for each specific $\mathrm{G} / \mathrm{D}$ and $\mathrm{RS}$, the maximum rate of the unsteadiness occurs near the lower cylinder.

- Regarding the local pressure distributions on the cylinders, it was found that rotating the cylinders shifts the front stagnation point in the clockwise direction for both cylinders as a function of RS. Additionally, the pressure distributions on the upper cylinder show higher sensitivity to the gap space between the cylinders in comparison to the lower cylinder. On the other hand, on the upper cylinder, the pressure difference between the half-outer and halfinner sides enhances as a function of $\mathrm{RS}$ at $\mathrm{G} / \mathrm{D}=3.0$ and at small and moderate G/D, this pressure difference attenuates at high RS. Regarding the lower cylinder, it was demonstrated that the pressure difference between the half-outer and half-inner sides enhances as a function of RS at whole under consideration $\mathrm{G} / \mathrm{D}$ values.
- Co-rotating two side-by-side cylinders provides a negative lift force on both cylinders as a function of $\mathrm{RS}$ at $\mathrm{G} / \mathrm{D}=3.0$. However, the magnitudes of the lift force at small and moderate G/D values reduces in the high RS values. In the case of the lower cylinder, the negative lift force enhances as a function of RS at all $\mathrm{G} / \mathrm{D}$ values.

- Distributions of the local Nusselt number over the upper cylinder showed that by co-rotating the cylinders, the rate of the heat transfer on the half-inner side enhances and reduces on the half-outer side up to $\mathrm{RS}=2$. However, at the higher RS values, a nearly uniform Nusselt number distribution was developed over the upper cylinder. Over the lower cylinder, the rate of the heat transfer enhances on the half-outer side and reduces on the half-inner side with $\mathrm{RS}$ and the nearly uniform distributions were seen at $\mathrm{RS}=4$ for all $\mathrm{G} / \mathrm{D}$ range.

- It was concluded that, at all G/D values, co-rotating two side-by-side cylinders reduces the heat transfer rate between the fluid flow and cylinders as a function of $\mathrm{RS}$ except the $\mathrm{G} / \mathrm{D}=2.0$ at which the maximum heat exchange rate of the lower cylinder occurs at $\mathrm{RS}=1$.

\section{Nomenclature}

Symbol Description

A area

$C_{D} \quad$ drag coefficient

$\bar{C}_{D} \quad$ mean drag coefficient

$C_{L} \quad$ lift coefficient

$\bar{C}_{L} \quad$ mean lift coefficient

$C_{p} \quad$ pressure coefficient

$c_{p} \quad$ specific pressure

$D \quad$ cylinder diameter

$F_{D} \quad$ drag force

$F_{L} \quad$ lift force

$G \quad$ gap space between the cylinders

$k$ conductivity

$n$ surface vertical vector

$\mathrm{Nu} \quad$ Nusselt number

$\overline{\mathrm{Nu}} \quad$ mean Nusselt number

$p \quad$ pressure

Pr Prandtl number $\left(=\frac{\mu c_{p}}{k}\right)$

$r \quad$ radial coordinate

$R \quad$ cylinder radius

Re Reynolds number $\left(=\frac{\rho U D}{k}\right)$

$R S$

$t$

$T$ non-dimensional rotating speed $\left(=\frac{\omega_{o} D}{2 U}\right)$

time

temperature

streamwise velocity

time-averaged streamwise velocity

free-stream velocity 


$\begin{array}{ll}u_{r m s} & \begin{array}{l}\text { root mean square of the streamwise velocity } \\ \text { velocity vector }\end{array} \\ v & \text { vertical velocity } \\ \bar{v} & \text { time-averaged vertical velocity } \\ v_{r m s} & \text { root mean square of the vertical velocity } \\ x & \text { streamwise dimension of coordinates } \\ y & \text { vertical dimension of coordinates }\end{array}$

\section{Greek symbols}

$\mu \quad$ dynamic viscosity of the fluid

$v$ kinematic viscosity of the fluid

$\rho$ density of the fluid

$\alpha$ angular location

$\omega_{o}$ rotating speed

$\omega$ vorticity

$\tau \quad$ cyclic period of vortex shedding

\author{
Subscripts \\ 1 upper cylinder \\ 2 lower cylinder \\ $s \quad$ surface of the cylinder \\ $\infty$ free-stream
}

\section{References}

[1] Lloyd T P and James M 2016 Large eddy simulations of a circular cylinder at Reynolds numbers surrounding the drag crisis. Applied Ocean Research 59: 676-686

[2] Yeon S M, Yang J and Stern F 2016 Large-eddy simulation of the flow past a circular cylinder at sub-to super-critical Reynolds numbers. Applied Ocean Research 59: 663-675

[3] Gao D L, Chen W L, Li H and Hu H 2017 Flow around a circular cylinder with slit. Experimental Thermal and Fluid Science 82: 287-301

[4] Gonçalves R T, Franzini G R, Rosetti G F, Meneghini J R and Fujarra A L C 2015 Flow around circular cylinders with very low aspect ratio. Journal of Fluids and Structures 54: 122-141

[5] Nguyen V T and Nguyen H H 2016 Detached eddy simulations of flow induced vibrations of circular cylinders at high Reynolds numbers. Journal of Fluids and Structures 63: 103-119

[6] Hsieh S C, Low Y M and Chiew Y M 2016 Flow characteristics around a circular cylinder subjected to vortex-induced vibration near a plane boundary. Journal of Fluids and Structures 65: 257-277

[7] Zhang K, Katsuchi H, Zhou D, Yamada H and Han Z 2016 Numerical study on the effect of shape modification to the flow around circular cylinders. Journal of Wind Engineering and Industrial Aerodynamics 152: 23-40

[8] Kim S, Alam M M and Maiti D K 2018 Wake and suppression of flow-induced vibration of a circular cylinder. Ocean Engineering 151: 298-307

[9] Aljure D E, Lehmkhul O, Rodríguez I and Oliva A 2017 Three dimensionality in the wake of the flow around a circular cylinder at Reynolds number 5000. Computers and Fluids 147: 102-118
[10] Chung M H 2016 Transverse vortex-induced vibration of spring-supported circular cylinder translating near a plane wall. European Journal of Mechanics-B/Fluids 55: 88-103

[11] Deylami H M, Amanifard N, Hosseininezhad S S and Dolati F 2017 Numerical investigation of the wake flow control past a circular cylinder with Electrohydrodynamic actuator. European Journal of Mechanics-B/Fluids 66: 71-80

[12] Wang C, Tang H, Duan F and Simon C M 2016 Control of wakes and vortex-induced vibrations of a single circular cylinder using synthetic jets. Journal of Fluids and Structures 60: 160-179

[13] Xu W, Ma Y, Cheng A and Yuan H 2018 Experimental investigation on multi-mode flow-induced vibrations of two long flexible cylinders in a tandem arrangement. International Journal of Mechanical Sciences 135: 261-278

[14] Li Z, Prsic M A, Ong M C and Khoo B C 2018 Large Eddy Simulations of flow around two circular cylinders in tandem in the vicinity of a plane wall at small gap ratios. Journal of Fluids and Structures 76: 251-271

[15] Huertas-Cerdeira C, Fan B and Gharib M 2018 Coupled motion of two side-by-side inverted flags. Journal of Fluids and Structures 76: 527-535

[16] Xu W, Cheng A, Ma Y and Gao X 2018 Multi-mode flowinduced vibrations of two side-by-side slender flexible cylinders in a uniform flow. Marine Structures 57: 219-236

[17] Shaaban M and Mohany A 2018 Flow-induced vibration of three unevenly spaced in-line cylinders in cross-flow. Journal of Fluids and Structures 76: 367-383

[18] Alam M M, Zheng Q, Derakhshandeh J F, Rehman S, Ji C and Zafar F 2018 On forces and phase lags between vortex sheddings from three tandem cylinders. International Journal of Heat and Fluid Flow 69: 117-135

[19] Chen W, Ji C, Williams J, Xu D, Yang L and Cui Y 2018 Vortex-induced vibrations of three tandem cylinders in laminar cross-flow: Vibration response and galloping mechanism. Journal of Fluids and Structures 78: 215-238

[20] da Silva B L, Luciano R D, Utzig J and Meier H F 2018 Flow patterns and turbulence effects in large cylinder arrays. International Journal of Heat and Fluid Flow 69: 136-149

[21] Alam M M, Zheng Q and Hourigan K 2017 The wake and thrust by four side-by-side cylinders at a low Re. Journal of Fluids and Structures 70: 131-144

[22] Shaafi K, Naik S N and Vengadesan S 2017 Effect of rotating cylinder on the wake-wall interactions. Ocean Engineering 139: 275-286

[23] Rana K, Manzoor S, Sheikh N A, Ali M and Ali H M 2017 Gust response of a rotating circular cylinder in the vortex suppression regime. International Journal of Heat and Mass Transfer 115: 763-776

[24] Bayat R and Rahimi A B 2017 Numerical solution of threedimensional NS equations and energy in the case of unsteady stagnation-point flow on a rotating vertical cylinder. International Journal of Thermal Sciences 118: 386-396

[25] Thakur P, Tiwari N and Chhabra R P 2018 Flow of a powerlaw fluid across a rotating cylinder in a confinement. Journal of Non-Newtonian Fluid Mechanics 251: 145-161.

[26] Naik S N, Vengadesan S and Prakash K A 2017 Numerical study of fluid flow past a rotating elliptic cylinder. Journal of Fluids and Structures 68: 15-31

[27] Hassanzadeh R 2018 Effects of Unsteady Flow Generation Over a Hot Plate on the Cooling Mechanism Using a 
Rotating Cylinder. Arabian Journal for Science and Engineering 1-11

[28] Darvishyadegari M and Hassanzadeh R 2018 Convective heat transfer and fluid flow of two counter-rotating cylinders in tandem arrangement. Acta Mechanica 229(4): 1783-1802

[29] Darvishyadegari M and Hassanzadeh R 2019 Heat and fluid flow around two co-rotating cylinders in tandem arrangement. International Journal of Thermal Sciences 135: 206-220

[30] Darvishyadegari M and Hassanzadeh R 2018 Analysis of the convective heat transfer and flow behavior around two counter-rotating side-by-side cylinders. Heat TransferAsian Research 47(6): 835-854

[31] Selimefendigil F, Ismael M A and Chamkha A J 2017 Mixed convection in superposed nanofluid and porous layers in square enclosure with inner rotating cylinder. International Journal of Mechanical Sciences 124: 95-108

[32] Kareem A K and Gao S 2017 Mixed convection heat transfer of turbulent flow in a three-dimensional lid-driven cavity with a rotating cylinder. International Journal of Heat and Mass Transfer. 112: 185-200

[33] Khanafer K and Aithal S M 2017 Mixed convection heat transfer in a lid-driven cavity with a rotating circular cylinder. International Communications in Heat and Mass Transfer 86: 131-142

[34] Selimefendigil F and Öztop H F 2018 Mixed convection of nanofluids in a three dimensional cavity with two adiabatic inner rotating cylinders. International Journal of Heat and Mass Transfer 117: 331-343
[35] Patankar S V 1980 Numerical Heat transfer and Fluid Flow, New York: Taylor \& Francis

[36] Ding H, Shu C, Yeo K S and Xu D 2007 Numerical simulation of flows around two circular cylinders by mesh-free least square-based finite difference methods. International Journal for Numerical Methods in Fluids 53(2): 305-332

[37] Sarvghad-Moghaddam H, Nooredin N and Ghadiri-Dehkordi B 2011 Numerical simulation of flow over two side-by-side circular cylinders. Journal of Hydrodynamics, Ser. B 23(6): 792-805

[38] Meneghini J R, Saltara F, Siqueira C L R and Ferrari Jr J A 2001 Numerical simulation of flow interference between two circular cylinders in tandem and side-by-side arrangements. Journal of Fluids and Structures 15(2): 327-350

[39] Hilpert R 1933 Heat transfer from cylinders. Forsch. Geb. Ingenieurwes 4: 215

[40] Churchill S W and Bernstein M 1977 A correlating equation for forced convection from gases and liquids to a circular cylinder in crossflow. Journal of Heat Transfer 99(2): 300-306

[41] Harimi I and Saghafian M 2012 Numerical simulation of fluid flow and forced convection heat transfer from tandem circular cylinders using overset grid method. Journal of Fluids and Structures 28: 309-327

[42] Hassanzadeh R, Sahin B and Ozgoren M 2013 Large eddy simulation of flow around two side-by-side spheres. Journal of Mechanical Science and Technology 27(7): 1971-1979 